\title{
A Global Climatology of Baroclinically Influenced Tropical Cyclogenesis*
}

\author{
RON MCTAGgaRT-COWAN \\ Numerical Weather Prediction Research Section, Environment Canada, Dorval, Quebec, Canada \\ THOMAS J. GALARNEAU JR. \\ National Center for Atmospheric Research, ${ }^{+}$Boulder, Colorado \\ LANCE F. BOSART \\ Department of Earth and Atmospheric Sciences, University at Albany, State University of New York, Albany, New York \\ RICHARD W. MOORE \\ Department of Meteorology, Naval Postgraduate School, Monterey, California \\ OLIVIA MARTIUS \\ Institute of Geography and Oeschger Centre for Climate Change Research, University of Bern, Bern, Switzerland
}

(Manuscript received 3 July 2012, in final form 15 November 2012)

\begin{abstract}
Tropical cyclogenesis is generally considered to occur in regions devoid of baroclinic structures; however, an appreciable number of tropical cyclones (TCs) form in baroclinic environments each year. A global climatology of these baroclinically influenced TC developments is presented in this study. An objective classification strategy is developed that focuses on the characteristics of the environmental state rather than on properties of the vortex, thus allowing for a pointwise "development pathway" classification of reanalysis data. The resulting climatology shows that variability within basins arises primarily as a result of local surface thermal contrasts and the positions of time-mean features on the subtropical tropopause. The pathway analyses are sampled to generate a global climatology of 1948-2010 TC developments classified by baroclinic influence: nonbaroclinic (70\%), low-level baroclinic (9\%), trough induced $(5 \%)$, weak tropical transition $(11 \%)$, and strong tropical transition (5\%). All basins other than the North Atlantic are dominated by nonbaroclinic events; however, there is extensive interbasin variability in secondary development pathways. Within each basin, subregions and time periods are identified in which the relative importance of the development pathways also differs. The efficiency of tropical cyclogenesis is found to be highly dependent on development pathway. The peak efficiency defined in the classification subspace straddles the nonbaroclinic/ trough-induced boundary, suggesting that the optimal environment for TC development includes a baroclinic contribution from an upper-level disturbance. By assessing the global distribution of baroclinically influenced TC formations, this study identifies regions and pathways whose further study could yield improvements in our understanding of this important subset of TC developments.
\end{abstract}

\footnotetext{
* Supplemental information related to this paper is available at the Journals Online website: http://dx.doi.org/10.1175/MWR-D12-00186.s1.

+ The National Center for Atmospheric Research is sponsored by the National Science Foundation.
}

Corresponding author address: Ron McTaggart-Cowan, 2121 Trans-Canada Highway, Floor 5, Dorval, QC H9P 1J3, Canada. E-mail: ron.mctaggart-cowan@ec.gc.ca

\section{Introduction}

The global tropical cyclone (TC; all acronyms defined in Table 1) climatology of Gray (1968) serves as a primary reference for TC development mechanisms and environmental properties associated with TC formation. While the majority of events included in the study were classified as "tropical" (type A, 83\%), the remainder were assigned to "subtropical" and "hybrid" categories. 
TABLE 1. List of acronyms used in this article.

\begin{tabular}{ll}
\hline \hline Acronym & \multicolumn{1}{c}{ Definition } \\
\hline IBTrACS & $\begin{array}{c}\text { International Best Track } \\
\text { Archive for Climate Stewardship } \\
\text { Linear discriminant analysis } \\
\text { LDA }\end{array}$ \\
LTM & Latent trajectory model \\
M08 & McTaggart-Cowan et al. (2008) \\
PDF & Probability density function \\
PV & Potential vorticity \\
PVU & Potential vorticity unit \\
QG & Quasigeostrophic \\
RWB & Rossby wave break \\
TC & Tropical cyclone \\
TT & Tropical transition \\
TUTT & Tropical upper-tropospheric trough \\
WMO & World Meteorological Organization \\
\hline
\end{tabular}

The dominance of type-A developments has led to a focusing of TC research on formations that follow this development pathway. However, the fact that $17 \%$ of events deviate from the tropical paradigm prompted increasing interest in the alternative subtypes of tropical cyclogenesis over the subsequent decades, especially once satellite and reanalysis data revealed a rich array of structure in the tropical upper atmosphere (Sadler 1976, 1978; Challa and Pfeffer 1980; Gray 1988; Bosart and Bartlo 1991; Montgomery and Shapiro 1993; Hess et al. 1995; Elsner et al. 1996).

The formulation of the weak and strong tropical transition (TT) paradigms by Davis and Bosart $(2003,2004)$ has reinvigorated interest in the possible influences of baroclinicity on TC formation. In the North Atlantic basin, Hulme and Martin (2009a,b) demonstrate similarities between TT-induced warm core development and the frontal occlusion process, and stress the importance of latent heat release on the evolution of the vortex. A climatology of North Atlantic subtropical developments presented by Evans and Guishard (2009) and Guishard et al. (2009) suggests that up to $33 \%$ of warm-season events may fall into this baroclinically influenced category. The increased emphasis on subtropical and hybrid storm formation led to the creation of a World Meteorological Organization (WMO) working group on the subject as part of the Seventh International Workshop on Tropical Cyclones (Rogers 2011).

In a reanalysis-based climatology of North Atlantic TC developments, McTaggart-Cowan et al. (2008, hereafter M08) show that less than half of storms form in nonbaroclinic environments, while the satellite-based climatology of Payne and Methven (2012) suggests that $63 \%$ of TC developments in the southern Indian Ocean occur in association with upper-level disturbances. The influence of such features in the Australian region is described by McBride and Keenan (1982) and in the west Pacific by Kelley and Mock (1982). In the latter, sustained near-surface convergence induced by quasigeostrophically (QG) forced ascent downshear of upper-tropospheric cold lows is shown to be capable of promoting TC development.

Expanding upon the results of M08, the primary goal of the current study is to create a coherent global climatology that documents baroclinic influences on TC formation. The resulting overview of baroclinic development environments in each basin will also serve as a basis for further research aimed at assessing the causal links between environmental baroclinicity and tropical cyclogenesis.

This study begins with a description of the datasets and classification techniques in section 2 , including a comparison with the results obtained by M08 for the North Atlantic basin. The climatological distribution of each of the five identified environmental categories is described in section 3. The corresponding TC development climatology is presented in section 4 , with an emphasis on comparison to previous regional climatologies and a basin-by-basin description of baroclinic influences on formation. The results of the preceding sections are combined in section 5, wherein an analysis of TC formation efficiency by development pathway is presented. The result of a transformation from geographical to classification space allows for further synthesis of the results in section 6 . The study concludes with a discussion of potential avenues for further research.

\section{Data and methodology}

The period considered in this study (1948-2010) is limited to $63 \mathrm{yr}$ by the availability of high-quality reanalysis and TC track data (Landsea et al. 2004). All discussions are based on this period unless otherwise noted.

\section{a. Classification metrics}

A pair of metrics were developed by M08 to represent the key characteristics of baroclinically influenced cyclogenetic pathways. The reduction of dimensionality afforded by the chosen metrics makes the classification problem tractable, yet enough information is retained to render the resulting categories physically meaningful. To focus on the role of environmental baroclinicity in TC development, the metrics are chosen to represent the background state rather than the structure of the vortex itself. The metrics are log normalized for subsequent statistical analyses as described by M08.

The $\mathbf{Q}$ metric is defined as the average convergence of the 400-200-hPa $\mathbf{Q}_{\text {nd }}$ vector within $6^{\circ}$ of the point of interest. Unlike the standard derivation of the $\mathbf{Q}$ vector 
following Hoskins et al. (1978), this metric uses the nondivergent wind in its formulation to eliminate problems associated with high Rossby number flows near the equator [Nielsen-Gammon and Gold (2008) and D. Keyser 2008, personal communication]:

$$
\mathbf{Q}_{\mathrm{nd}}=-\frac{R}{\sigma p}\left(\begin{array}{l}
\frac{\partial \mathbf{v}_{\mathrm{nd}}}{\partial x} \cdot \nabla_{p} T \\
\frac{\partial \mathbf{v}_{\mathrm{nd}}}{\partial y} \cdot \nabla_{p} T
\end{array}\right)
$$

Here, $\mathbf{v}_{\mathrm{nd}}$ is the vector nondivergent wind, $T$ is the drybulb temperature, $p$ is the atmospheric pressure, $\sigma$ is the dry static stability, $R$ is the gas constant for dry air, and $\nabla_{p}$ is the gradient operator applied on an isobaric surface. As described by M08, $\mathbf{Q}$ metric represents synoptic-scale forcing for ascent, usually provided by an upper-level trough. Sustained dynamically forced ascent leads to moistening of the column as the temperature profile destabilizes toward static neutrality. Lower-level convergence in the trough-induced ascent region creates an area of enhanced cyclonic relative vorticity that can serve as a seedling for further axisymmetrization (Ritchie and Holland 1993). Additionally, convection is favored in the region of large-scale ascent, leading to enhanced organization of convective elements and the intensification of the lower-level circulation (Simpson et al. 1997).

The lower-level thickness asymmetry (Th) is defined as the maximum difference between the $1000-700-\mathrm{hPa}$ thickness values in two hemispheres (semicircles) within $10^{\circ}$ of the point of interest. Low-level baroclinicity is an important ingredient of the Davis and Bosart (2004) TT paradigm, wherein isentropic ascent across the remnant baroclinic zones of the precursor vortex focuses on convective activity. An elevated value of the Th metric may also be associated with a low-level jet (Findlater 1969; Karyampudi and Carlson 1988). The role that such thermal contrasts play in TC development is a subject of active debate in the literature (Karyampudi and Pierce 2002; Dunion and Velden 2004; Braun 2010).

\section{b. Gridded and TC datasets}

The classification technique used in this study, described in section $2 \mathrm{c}$ and the appendix, benefits from the use of an ensemble of reanalysis data. Following M08, the National Centers for Environmental Prediction-National Center for Atmospheric Research Reanalysis I (Kalnay et al. 1996) serves as the baseline gridded dataset since it covers the full period of interest. All of the other pressurelevel reanalysis products are interpolated or aggregated to the $2.5^{\circ}$ Reanalysis-I grid. From the European Centre for Medium-Range Weather Forecasts (ECMWF), both the 40-yr ECMWF Re-Analysis [ERA-40, 1957-2002; Uppala et al. (2005)] and the ECMWF Re-Analysis Interim [ERA-Interim, 1989-present; Dee et al. (2011)] are employed. The Japan Meteorological Agency Reanalysis (JRA-25) grids are used over their full period of availability, which covers 1979-present (Onogi et al. 2007).

Values of the $\mathbf{Q}$ and Th metrics are computed for each of the reanalyses. These allow for calculation of both the mean and the unbiased variance of the metrics, hereafter referred to as the "combined reanalysis" dataset. The aggregation and averaging steps reduce the fidelity of the vortex representation (Schenkel and Hart 2012); however, the metrics are designed to represent the environmental structure instead. Mean values are used for classification as described in the following section, while variances serve as inputs to the classification quality estimate outlined in the appendix.

The International Best Track Archive for Climate Stewardship (IBTrACS) dataset is a WMO-endorsed synthesis of available global TC tracking data (Knapp et al. 2010). The IBTrACS basin definitions are employed throughout this study for consistency. These basins, along with ocean region names and currents mentioned in this study, are shown in Fig. 1 for reference. The v03r03 edition of IBTrACS includes TC tracking information from non-WMO sources. Storms identified only by those sources are classified so that development pathway information is available for future investigations; however, they are not considered during the analysis presented here.

Throughout this study, the initial time $T_{0}$ for each TC is defined to be the first report time in the IBTrACS archive. The lack of consistent wind reporting across basins, and large discrepancies in wind estimates between forecast centers (Knapp et al. 2010) makes a global windbased estimate of $T_{0}$ impractical. Instead, the chosen time represents the first time at which regional specialists determine that the vortex is sufficiently organized and intense to be tracked. To eliminate storms whose initial development stages are not captured in the best track, any TCs with $T_{0}$ winds exceeding the 34-kt $\left(\sim 17.5 \mathrm{~m} \mathrm{~s}^{-1}\right)$ tropical storm threshold are not considered. This is consistent with the definition of $T_{0}$ employed by Elsner et al. (1996) and M08 using the Atlantic basin hurricane database (HURDAT; Landsea et al. 2004). An extensive set of sensitivity tests have been undertaken with different definitions of $T_{0}$, all of which produce qualitatively identical results. This suggests that any errors associated with $T_{0}$ selection are random in nature, and do not bias classifications toward any particular pathway. Track data from HURDAT will be used here only for the reclassification comparison presented at the end of section 2c. 


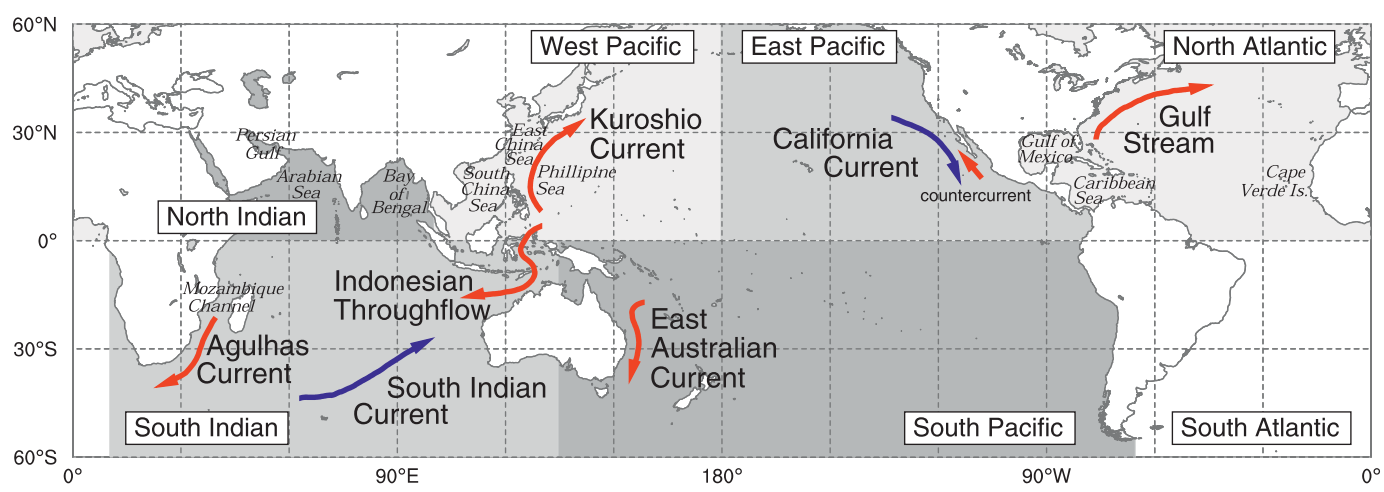

FIG. 1. Ocean currents, regions, and basins mentioned in the text. Surface currents are identified as either warm (red) or cold (blue) with the direction of flow indicated by an arrowhead. Regions are labeled with italicized text, and basins (as defined in the IBTrACS dataset) are gray shaded and labeled.

\section{c. Pathway classifications}

A latent trajectory model [LTM; Lazarsfeld and Henry (1968)] was used by M08 to create an optimal set of TC development classes (also called "pathways") based on trajectories through metric space between $T_{0-36 \mathrm{~h}}$ and $T_{0}$. The use of vortex back tracking in the calculation and the latency of the technique, make it illsuited for extensions to environmental classifications (without a TC present), new time periods, and other basins. Instead, a linear discriminant analysis [LDA; Friedman (1989)] is employed here at $T_{0-12 \mathrm{~h}}$, using the classifications made by M08 as the training dataset. This analysis time is chosen because it is found to yield a slightly higher correct reclassification rate than other times; however, the results presented here are qualitatively insensitive to this choice. All resulting objectively identified category names are italicized herein to distinguish them from text. A detailed discussion of the LDA technique is available in the appendix.

M08 identify six TC development pathways in their study of 1948-2004 North Atlantic formations. A nonbaroclinic pathway that conforms to the standard TC development model is followed by $40 \%$ of storms, primarily in the main development region (Goldenberg and Shapiro 1995) and the Gulf of Mexico. A further
$13 \%$ of TCs form in environments characterized as lowlevel baroclinic, largely near the Cape Verde Islands and in association with the African easterly jet (Burpee 1972). Trough-induced formations account for just $3 \%$ of storms in the M08 climatology, and tend to occur near the Gulf of Mexico early in the hurricane season. The weak TT and strong TT pathways described by Davis and Bosart (2003) are followed by $13 \%$ and $15 \%$ of North Atlantic TCs, respectively, and are associated with remnant circulations and baroclinic zones of midlatitude origin. A brief description of each of these pathways is provided in Table 2 for reference. A rapidly evolving pathway in which an upper-level disturbance temporarily enhances the $\mathbf{Q}$ metric during development is identified by M08 as transient trough interaction $(16 \%$ of cases, primarily in the Gulf of Mexico). The instantaneous nature of the LDA technique means that TCs in this category must be reassigned into the troughinduced, weak TT, and strong TT classes for the purposes of this study. A description of the reassignment procedure is included in the appendix.

A contingency table of the LDA-based reclassification of the original 496-storm climatology of M08 is shown as Table 3. Given an $84 \%$ correct-reclassification rate (Fig. 2) and the skill of the LDA-based classifications for the subjectively analyzed 18-case sample presented by

TABLE 2. Description of the TC development pathways identified in this study. The short name of each category (first column) will be used to describe the pathway throughout the text. This is essentially a tabular representation of Fig. 8 of M08.

\begin{tabular}{|c|c|c|c|}
\hline \multirow[b]{2}{*}{ Category (pathway) } & \multicolumn{2}{|c|}{ Metric value } & \multirow[b]{2}{*}{ Brief description } \\
\hline & $\mathbf{Q}$ & Th & \\
\hline Nonbaroclinic & Low & Low & No appreciable baroclinic influences \\
\hline Low-level baroclinic & Low & High & Strong lower-level thermal gradients without an upper-level disturbance \\
\hline Trough induced & High & Low & Upper-level disturbance without appreciable lower-level thermal gradients \\
\hline Weak TT & High & Medium & Upper-level disturbance with moderate lower-level thermal gradients \\
\hline Strong TT & High & High & Upper-level disturbance with strong lower-level thermal gradients \\
\hline
\end{tabular}


TABLE 3. Reclassification contingency table comparing the 496 regrouped LTM classifications of M08 (columns) to the North Atlantic LDA classifications (rows). Values are shown as TC development counts, and correct reclassifications on the diagonal are shown in bold.

\begin{tabular}{|c|c|c|c|c|c|c|}
\hline & & \multicolumn{5}{|c|}{ LTM classification } \\
\hline & & Nonbaroclinic & Low-level baroclinic & Trough induced & Weak TT & Strong TT \\
\hline \multirow[t]{5}{*}{ LDA classification } & Nonbaroclinic & 184 & 3 & 9 & 14 & 0 \\
\hline & Low-level baroclinic & 2 & 55 & 0 & 0 & 3 \\
\hline & Trough induced & 4 & 0 & 21 & 5 & 0 \\
\hline & Weak TT & 10 & 2 & 6 & 85 & 15 \\
\hline & Strong TT & 0 & 3 & 0 & 5 & 70 \\
\hline
\end{tabular}

M08 (14 correct using the LTM and 13 correct using the LDA, see the online supplement to this article for details), the LDA is considered to yield results of sufficient quality to allow it to serve as the primary classification tool for the remainder of this study.

\section{d. Terminology}

The discussions throughout this study will be undertaken from a potential vorticity (PV) perspective (Hoskins et al. 1985). The terminology associated with this diagnostic approach is not inconsistent with that of previous investigations of baroclinic influences on the tropical environment. Since localized departures from a basic state are fundamental ingredients of PV-based diagnosis, all PV anomalies described hereafter are defined with respect to the zonal mean.

The tropical upper-tropospheric troughs (TUTTs) described by Riehl (1948) and Sadler (1967, 1976, 1978) are time-mean features of the summer state in the midocean regions, represented by the equatorward penetration of a lowered subtropical tropopause. From a thermodynamic perspective, the formation and maintenance of the TUTTs can be explained by radiatively induced subsidence driven by sensible and latent heating contrasts between the continents and adjacent oceans (Fitzpatrick et al. 1995). Dynamically, the midocean regions are shown to be active areas of summer anticyclonic Rossby wave breaking (RWB) by Postel and Hitchman (1999) and Ndarana and Waugh (2011). These characterizations of the troughs are not contradictory, since the dominant anticyclonic PV anomaly displaced poleward during anticyclonic RWB is necessarily eroded by radiative processes, leading to net radiational cooling in the midocean regions. For consistency with Postel and Hitchman (1999), the 350-K isentrope on the 2-PV unit (PVU, where $1 \mathrm{PVU}=1 \times 10^{-6} \mathrm{~m}^{2} \mathrm{~s}^{-1} \mathrm{~K} \mathrm{~kg}^{-1}$ ) surface is used to indicate the position of the subtropical tropopause in this study. Hereafter, the traditional TUTT terminology will be used to describe these baroclinic midocean trough features whose formation and maintenance mechanisms are understood to comprise both thermodynamic (diabatic heating) and dynamic (RWB) components.
Isolated cyclonic PV anomalies that originate within the midocean troughs have been referred to as TUTT cells (Colton 1973), upper cold lows (Erickson 1971), upper-tropospheric cyclonic vortices (Kousky and Gan 1981), and variants of these names. These features will be discussed in more detail later in the following section, but are generally formed by the breakdown of PV streamers following RWB near the subtropical tropopause (Appenzeller and Davies 1992; Sakamoto and Takahashi 2005; Martius et al. 2007).

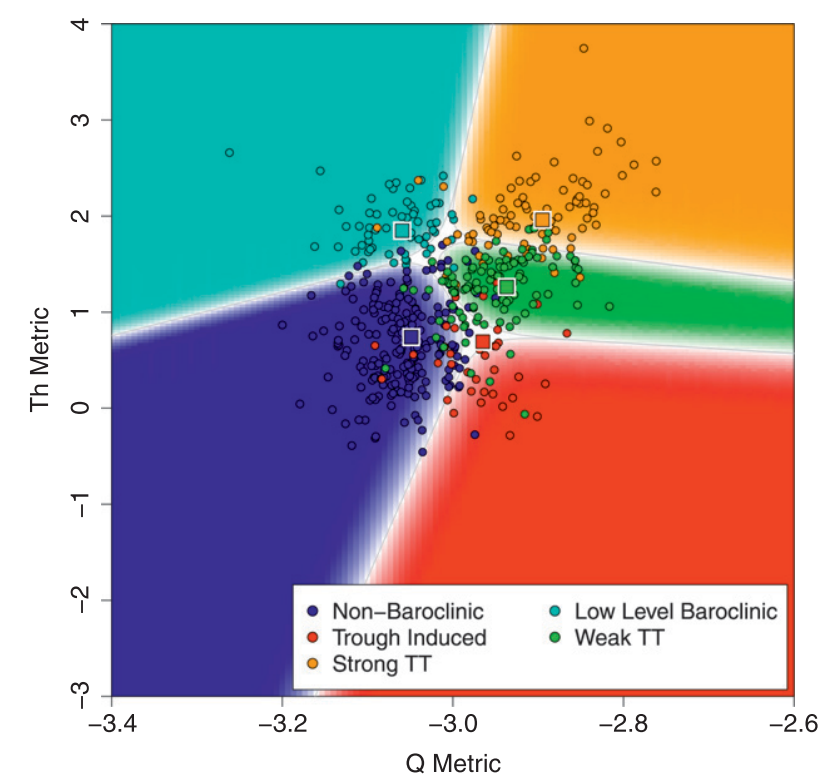

FIG. 2. Reclassification of North Atlantic TC pathways studied by M08 in metric space. Each of the 496 events is plotted at the position in the $\mathbf{Q}-$ Th plane given by its metric values. The event dots are color coded according to the LTM class diagnosed by M08 as indicated in the legend. The same color scheme is used for the LDA-based background, which represents the dominant pathway in each sector of metric space scaled to white with decreasing posteriors (see the appendix for details). When a dot lies on the background of the same color, the LDA reclassification reproduces the original LTM result. The thin gray lines represent the $50 \%$ posterior for each class, and the centroids of the classes are shown by color-coded squares in each panel. 

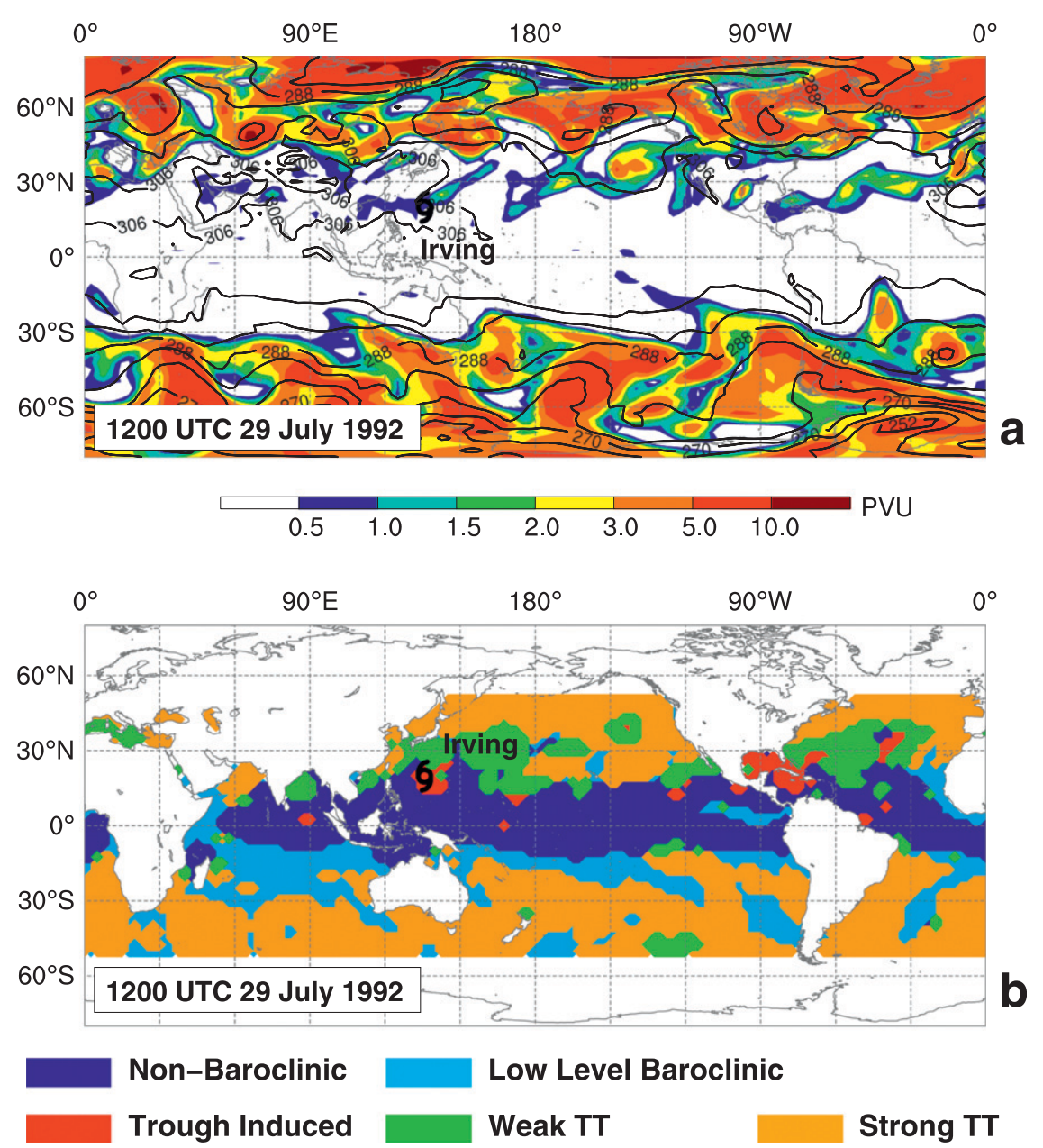

FIG. 3. (a) Layer-mean 400-200-hPa PV (shaded in PVU as indicated on the color bar, where $1 \mathrm{PVU}=1 \times 10^{-6} \mathrm{~m}^{2} \mathrm{~s}^{-1} \mathrm{~K} \mathrm{~kg}^{-1}$ ) and 1000-700-hPa thickness (solid lines at 6-dam intervals) from the ERA-Interim for 1200 UTC 29 Jul 1992. (b) The LDA-based pathway classifications for the combined reanalyses valid at the same time. In both (a) and (b), the formation position of Typhoon Irving at 0000 UTC $30 \mathrm{Jul}$ is indicated with a tropical storm symbol.

\section{Pathway climatology}

The LDA-based classification scheme can be applied globally at each analysis time since it does not rely on the presence of a TC for pathway selection. This allows for the identification of a preferred development category at each point on the combined reanalysis grid that is indicative of the pathway that a TC forming in the region over the subsequent 12 -h period would follow. A development pathway climatology can therefore be developed for all basins over the full 1948-2010 period.

An example of a gridded classification is provided in Fig. 3 for the analysis time corresponding to $T_{0-12 \mathrm{~h}}$ for Typhoon Irving (1992) in the Philippine Sea (Fig. 1). The TUTT cell implicated in Irving's development by Ritchie and Holland (1997) is represented by a fractured upper-level PV feature in Fig. 3a, above a region of weak thickness gradients. As a result, the trough-induced pathway is selected by the LDA as shown in Fig. 3b. A similar PV anomaly leads to a trough-induced region in the Gulf of Mexico. To the northeast of each of these areas, increasing baroclinicity results in weak TT classifications. Still farther north, midlatitude troughs create large areas of strong TT classification; however, TC development at these latitudes is unlikely because of strong vertical shears and low SSTs. Baroclinicity along the equatorial African coastlines leads to regions of lowlevel baroclinic classification, as do thickness gradients north of the westerlies in the winter Southern Hemisphere. The absence of TUTTs in the winter hemisphere is evinced by the lack of weak TT regions south of the equator. Elsewhere in the tropics, the nonbaroclinic pathway prevails as it does throughout much of the season in each basin. 
The climatological frequency of occurrence of each pathway is shown for three-month "early" and "late" summer seasons in Fig. 4. In the Northern Hemisphere, early summer extends from May to July, and late summer from August to October. The Southern Hemisphere early and late summer periods consist of NovemberJanuary and February-April, respectively. Monthly variability within these seasons is limited. Environments conducive to nonbaroclinic development modes dominate within $20^{\circ}$ of the equator across most of the world's oceans in both seasons (Figs. 4a,b). Local minima with respect to the zonal mean frequencies occur to the east of the TUTTs. In these regions, upper-level disturbances caused by breaking Rossby waves on the subtropical tropopause (Postel and Hitchman 1999) lead to environments that are inconsistent with the nonbaroclinic pathway.

Within $15^{\circ}$ of the equator, it is only in the Arabian Sea and near the Cape Verde Islands that the frequency of occurrence of the nonbaroclinic pathway falls below $10 \%$. Lower-level thermal contrasts play a dominant role in controlling the local environment in both these regions (Figs. 4c,d). As described by M08, baroclinicity beneath the African easterly jet (Burpee 1972) is associated with the enhanced frequency of this pathway over the eastern North Atlantic. Along the East African highlands, strong cross-equatorial flow leads to the formation of the southwesterly low-level Somali jet (Findlater 1969). This baroclinic jet extends from the African coast to the Bay of Bengal prior to the onset of the Indian summer monsoon (Boos and Emanuel 2009), leading to frequent low-level baroclinic environments across the north Indian Ocean (Fig. 4c). The postmonsoon season is characterized by a weaker jet (Findlater 1969) and the restriction of this pathway to the northeastern Arabian Sea as shown in Fig. 4d.

The unique configuration of the south Indian Basin leads to high frequencies of low-level baroclinic classification in a band extending from northwestern Australia into the center of the basin, most notable in early summer (Figs. 4c,d). The lack of land-sea thermal contrasts in the area suggests that SST gradients may be responsible for establishing background baroclinicity. Equatorward of this region, the Indonesian Throughflow transports heat from the warmest tropical ocean, the equatorial west Pacific, into the north branch of the south Indian subtropical gyre (Godfrey 1996). The south Indian Current, the poleward branch of the gyre, advects relatively cool water northeastward across the basin (Stramma 1992). While the bulk of upper-ocean baroclinicity is concentrated in the subtropical front south of $30^{\circ} \mathrm{S}$ (Belkin and Gordon 1996), Palastanga et al. (2007) show that strengthened meridional SST gradients extend equatorward in the eastern half of the basin, consistent with the prevalence of low-level baroclinic environments in the region.

The trough-induced pathway is the least frequently occurring of the environments diagnosed in this study. In the Northern Hemisphere, local maxima are apparent in the western North Pacific, the Gulf of Mexico, and the Caribbean, particularly during late summer (Figs. 4e,f). In the Southern Hemisphere, a single peak in troughinduced frequency is evident near the equator in the central South Pacific. These regions lie equatorward and westward of the primary subtropical RWB centers (Postel and Hitchman 1999; Ndarana and Waugh 2011), identifiable in Fig. 4 as areas in which the separation between the plotted isentropes increases. During anticyclonic RWB, filaments of high-PV air are extruded from the stratospheric source and break down to form isolated cyclonic PV anomalies that are advected westward by the tropical easterlies (Sadler 1975; Kelley and Mock 1982; Chen and Chou 1994; Ferreira and Schubert 1999; Sakamoto and Takahashi 2005), leading to localized increases in the frequency of trough-induced environments.

The position and strong positive tilt of the weak TT maxima (Figs. 4g,h) coincide with the TUTT axes. Weak TT maxima are displaced poleward and eastward of their trough-induced counterparts (cf. Figs. 4e,f and 4g,h), consistent with the need for the moderate background baroclinicity that serves to discriminate between these pathways. This baroclinicity may arise from one of several sources: subtropical atmospheric fronts (Todd and Washington 1999) such as those that are uniformly present in the Southern Hemisphere summer at $30^{\circ} \mathrm{S}$ (Simmonds et al. 2012), precursor baroclinic vortices (Davis and Bosart 2002), or meridional SST gradients that exist poleward of the equatorial SST maxima (Reynolds et al. 2002).

At higher latitudes, the strong TT pathway dominates the climatology as a result of Rossby wave activity in the midlatitude westerlies (Figs. 4i,j). The equatorward displacement of strong TT frequency at the eastern boundaries of the North Pacific and North Atlantic basins is consistent with enhanced RWB at the exits of the midlatitude 330-K waveguides as shown by Martius et al. (2008). The strong TT frequency is more zonally uniform in the Southern Hemisphere although the presence of the polar front jet south of the South Atlantic basin and south Indian Basin reduces Rossby wave activity in the region, particularly in the early summer season (Ndarana and Waugh 2011). By the late summer, wave breaking on the $330-\mathrm{K}$ isentropic surface occurs across most of the southern oceans, leading to a zonal expansion of the high-frequency ( $>75 \%$ ) strong TT region as shown in Fig. 4j. 


\section{Early Summer}
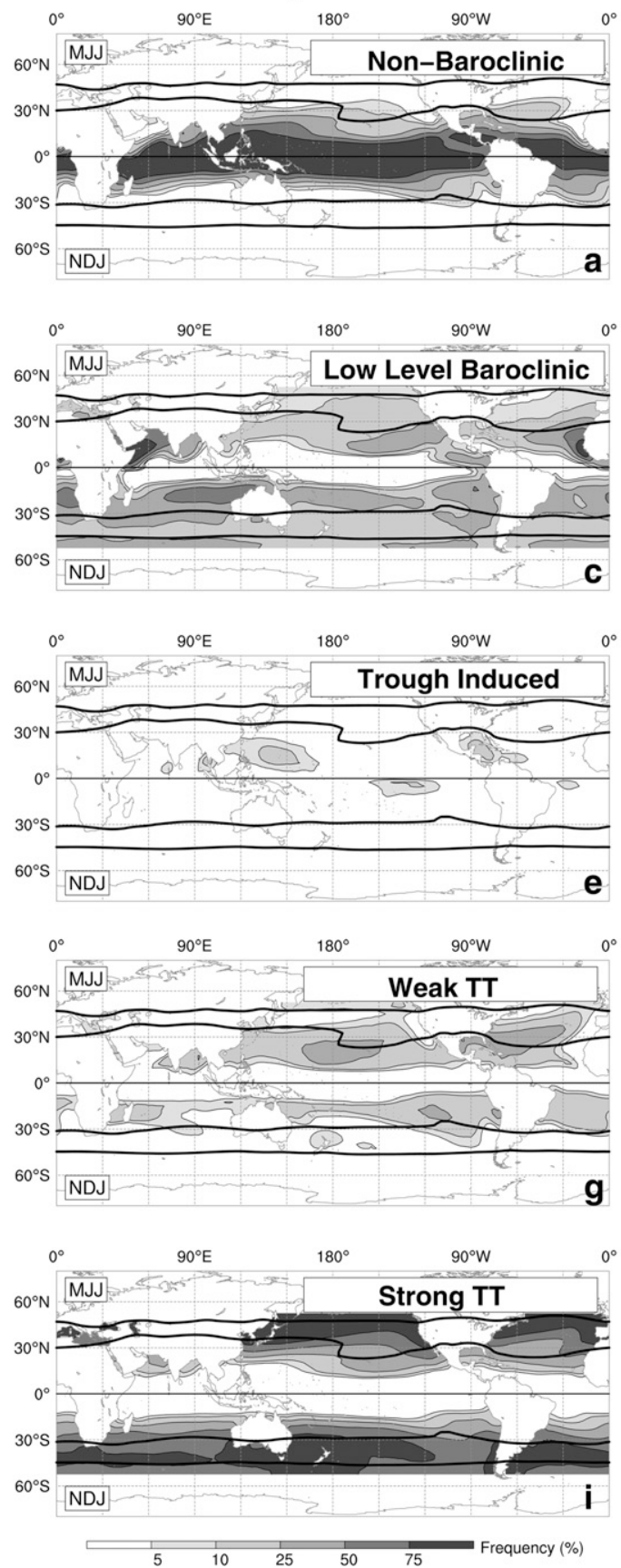

\section{Late Summer}
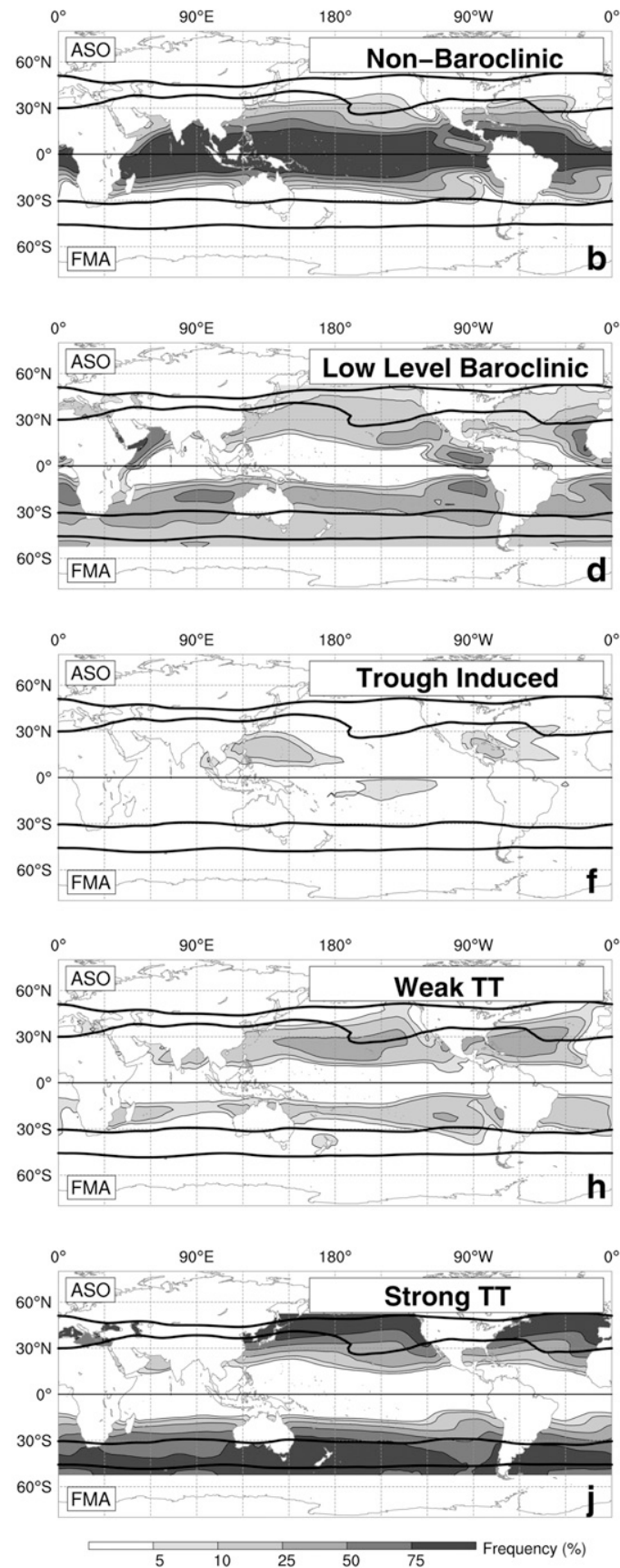

FIG. 4. Frequency of occurrence (in percentage as indicated on the grayscale bar) of pathways in the climatology for the (a),(c),(e),(g),(i) early summer and (b),(d),(f),(h),(j) late summer in each hemisphere. The pathways are ordered in rows, as indicated in the top-right corner of each panel. The three months associated with a season in each hemisphere are identified on the left-hand side of each panel with the first letters of the month names. The seasonalmean 2-PVU contour on the 350- (equatorward) and 330-K (poleward) surfaces are plotted with heavy solid lines to provide a reference for subtropical and midlatitude tropopause structures, respectively. A thin solid line is drawn along the equator to represent the hemispheric temporal discontinuity of the plots. 


\section{Global TC classification}

The pathway analyses described in the previous section are sampled at $T_{0-12 \mathrm{~h}}$ for each TC in the 1948-2010 period that meets the conditions outlined in section $2 \mathrm{~b}$. To assess the reliability of the resulting development pathway climatology, the classifications are compared with results from previous investigations of TC development types in as many basins as possible. In each case, the documented selection criteria are employed; however, use of the recently compiled IBTrACS dataset leads to minor inconsistencies in event counts. Following these climatological comparisons, an analysis of development pathways by basin is undertaken with an understanding of the strengths and potential limitations of the current methodology.

\section{a. Comparison with previous climatologies}

Using a global climatology, Gray (1968) found that $83 \%$ of disturbances developed without being influenced by "broadscale baroclinic processes" (here assumed to be associated with the lower-level thickness gradients characterized by elevated Th values), a classification based on the position of the incipient vortex equatorward of $20^{\circ}$ latitude. Developments following a recombination of the nonbaroclinic and trough-induced classes account for $75 \%$ of global TC formations. Although some of the discrepancy between these results may be attributed to the difference in classification strategy, the lack of upper-air and satellite data over the majority of the Gray (1968) study period may have resulted in some underrepresentation of upper-level baroclinic influences on development (Gray 1988).

A synthesis of the LDA-classified events into the "tropical only" and "baroclinically influenced" classes used by Hess et al. (1995) and Elsner et al. (1996) for their studies of North Atlantic hurricanes proceeds using the nonbaroclinic category for the former, and all remaining categories for the latter. The result of this recombination for the $1950-93$ period is $49 \%$ tropicalonly (99/199) and 51\% baroclinically influenced developments (100/199). This compares well with the Elsner et al. (1996) result of with $46 \%$ and $54 \%$, respectively. The comparison of a sample of individual classifications is provided in the online supplement to this article, and shows $75 \%$ classification agreement for the full period, with no evidence of a systematic error mode.

The influence of synoptic-scale perturbations on TC development in the active western North Pacific basin is documented by Briegel and Frank (1997) using a short climatology (1988-89, 41 cases). An upper-level trough lies within $1000 \mathrm{~km}$ of the developing center in $22 \%$
(9/41) of TC formation events. The authors suggest that large-scale uplifting in advance of the trough axis makes such a configuration favorable for development in the region. The subjective case selection criteria of the original investigation are merged with those of the current study to identify all possible matching events. Of the resulting 44-member sample, 12 cases $(27 \%)$ are identified as trough-induced, weak TT, or strong TT events. This is consistent with the results of Briegel and Frank (1997) and suggests that the extension of the classification technique to the west Pacific basin produces reasonable results.

For the Australian region $\left(5^{\circ}-30^{\circ} \mathrm{S}, 105^{\circ}-165^{\circ} \mathrm{E}\right)$, McBride and Keenan (1982) develop a detailed climatology of synoptic structures associated with 1974-79 TC developments. Strong westerly troughs of midlatitude origin are found to be present in five cases $(12 \%)$. The fact that three (7\%) trough-induced, weak TT, and strong TT events that are identified in the current climatology for a similarly defined subsample of cases suggests that the LDA-based classification produces results that are consistent with those of previous regional investigations.

In a study of TC development in the south Indian Ocean, Payne and Methven (2012) demonstrate the importance of baroclinic waves to cyclogenesis in the region. Using water vapor imagery available between 2001 and 2009, the authors identify 19 baroclinic wave events associated with 41 TC developments, representing $29 \%$ of the basin total (41/141). Over the same period, the current climatology suggests that trough-induced, weak TT, and strong TT events constitute $23 \%$ of TC developments in the region (31/134). The 2008 triple-development case study presented by Payne and Methven (2012) allows for a confirmation of coherency between the diagnostic approaches: 17S, Hondo, and Ivan are classified as weak TT, trough induced, and trough induced, respectively, in the current climatology.

The consistency of the pathway classifications with the results of previous climatological studies is encouraging, but a case-by-case evaluation of the diagnosed pathways is needed to provide an estimate of the accuracy of individual classifications. A set of 99 satellite-era TC developments have been subjectively classified based on their descriptions in the published literature. (A complete list of the selected storms and relevant references are provided in the online supplement to this article.) Overall, $78 \%$ of classifications match their subjective counterparts, although this quantity varies between the classes as shown in Table 4. The nonbaroclinic and strong TT categories have the highest correct classification rates, likely because they lie at the extrema of the phase space diagonal. There are fewer clear examples of the other pathway classes in the literature. Events that 
TABLE 4. Contingency table comparing 99 cases from the published literature (details provided in the online supplement), each subjectively assigned to a development pathway based on the case description (columns), to the LDA classification (rows). Values are shown as TC development counts, and correct classifications on the diagonal are shown in bold.

\begin{tabular}{|c|c|c|c|c|c|c|}
\hline & & \multicolumn{5}{|c|}{ Subjective classification } \\
\hline & & Nonbaroclinic & Low-level baroclinic & Trough induced & Weak TT & Strong TT \\
\hline \multirow[t]{5}{*}{ LDA classification } & Nonbaroclinic & 58 & 2 & 5 & 2 & 0 \\
\hline & Low-level baroclinic & 3 & 4 & 0 & 0 & 0 \\
\hline & Trough induced & 0 & 0 & 3 & 1 & 0 \\
\hline & Weak TT & 2 & 2 & 0 & 5 & 2 \\
\hline & Strong TT & 0 & 1 & 0 & 2 & 7 \\
\hline
\end{tabular}

are subjectively identified as low-level baroclinic are instead classified as weak TT or strong TT if an existing upper-level disturbance was not noted in the original study. Conversely, trough-induced events in the literature are classified as nonbaroclinic if the equatorward extension of an upper-level trough is not well represented in the combined reanalysis.

A classification "quality" attribute is assigned to each development event as described in the appendix. Figure 5 shows the ability of this measure to assess the confidence of the classification. Consideration of only highconfidence events (quality values greater than 0.6) can improve the correct-classification rate to $85 \%-90 \%$. The quality attribute will be particularly useful for composite and process studies, where only the clearly categorized events with a reliable representation in the reanalyses should be considered.

\section{b. TC development climatology}

The relative frequency of TC developments in each basin that follows a specific development pathway is shown in Fig. 6. Despite the change in dataset and methodology, the North Atlantic basin displays a similar breakdown of development pathway frequency to that described by M08 (cf. Fig. 9 and Table 3 of M08). The nonbaroclinic pathway dominates in all basins, although the secondary cyclogenetic pathway varies by region as shown in Fig. 7. In this section, the important development pathways in each basin are described. Whenever possible, current conceptual models for the region are used to describe the processes behind baroclinically influenced TC development following each pathway. When such a model does not exist, a development mechanism is proposed.

\section{1) North AtLANTiC BAsin}

The climatology of TC development pathways in the North Atlantic basin has been described by M08. Nonbaroclinic developments (Fig. 7b) occur primarily in the main development region and the Gulf of Mexico, with a peak frequency in September (Fig. 8b). Baroclinicity beneath the African easterly jet is an important ingredient in easterly wave developments near the Cape Verde islands, classified as low-level baroclinic events in this climatology (Norquist et al. 1977). Trough-induced and weak TT developments tend to occur in the Gulf of Mexico and extreme western Atlantic (Figs. 7d,e), with little seasonality between June and October in association with midlatitude troughs digging equatorward east of the Rocky Mountains (Figs. 8d,e). Over the western and central Atlantic, strong TT developments have occurred in all months except February and March, and increase in relative importance in the spring and fall (Figs. 7f and 8f). As described by Davis and Bosart (2003), these events rely on the favorable phasing of a baroclinic lower-level precursor over warm SSTs and a strong upper-level trough in the westerlies, a synoptic arrangement that is most frequently encountered in the shoulders of the Atlantic hurricane season near the Gulf Stream.

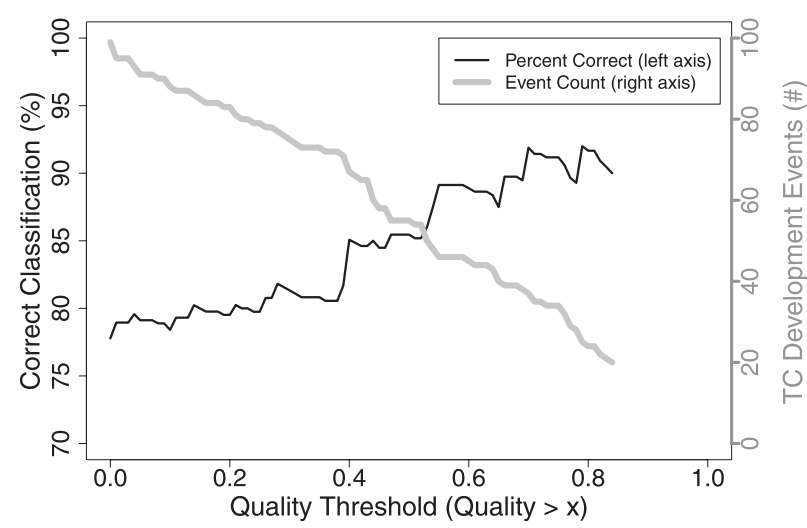

FIG. 5. Performance assessment of the classification quality attribute. The percentage of correct classifications (based on the 99 cases from the published literature described in the appendix) with quality values greater than the threshold on the abscissa is shown in the black line and corresponds to the left-hand axis. The number of storms that meet the given quality threshold is shown in the gray line and corresponds to the right-hand axis. Plotting only occurs for thresholds that yield at least 20 cases (quality $\leq 0.83$ ) to avoid instability at small sample sizes. 


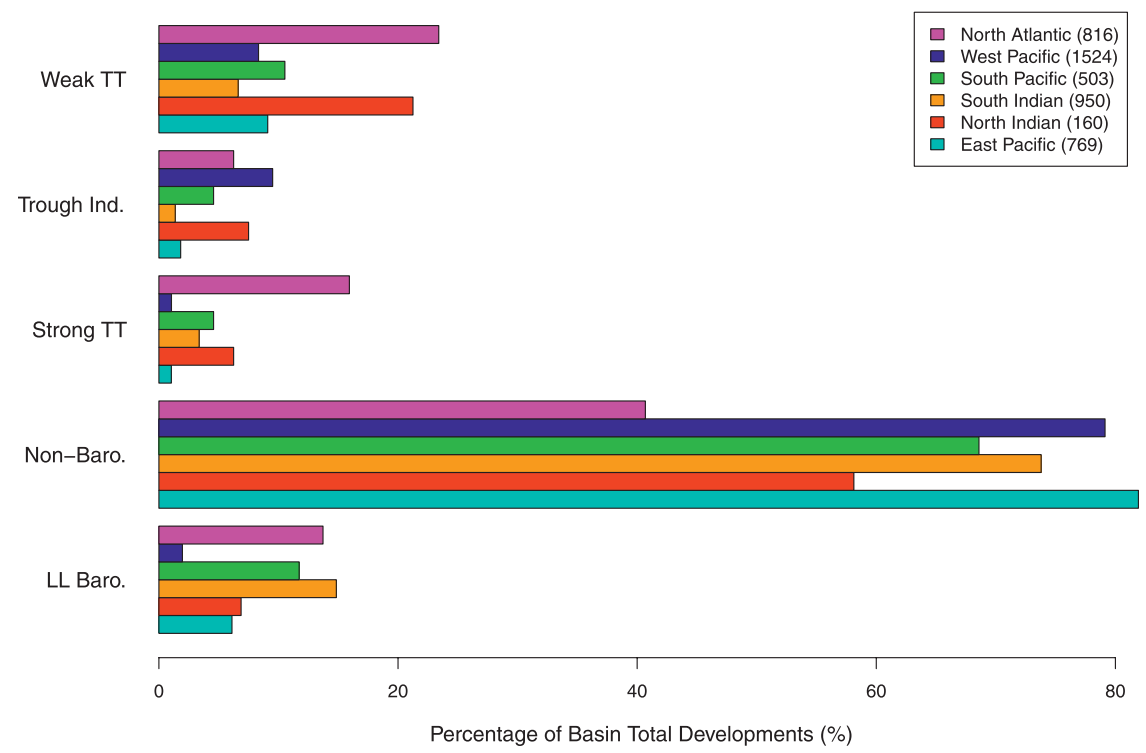

FIG. 6. Relative frequency of TC development following a specified pathway (vertical axis) by basin (colors as indicated on the legend). The number of storms meeting the climatology criteria in each basin is provided in the legend.

\section{2) EAST PACIFIC BASIN}

Nonbaroclinic developments are most dominant in the east Pacific basin, where over $80 \%$ of TCs follow this pathway (Figs. 6 and 7b). The July-August peak in nonbaroclinic frequency shown in Fig. 9b is earlier than the August-September peaks in low-level baroclinic and weak TT cyclogenesis frequencies shown in Figs. 9c,e. Although these baroclinically influenced events compose just $15 \%$ of total developments in the basin, they
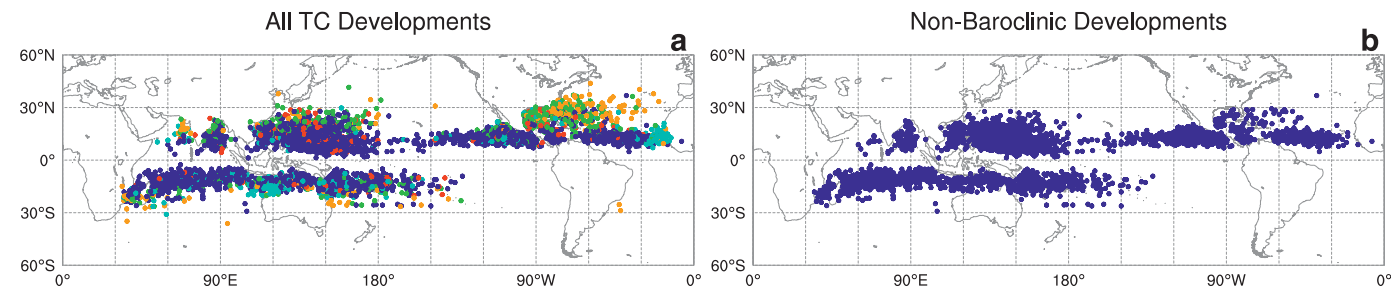

Low Level Baroclinic Developments

Trough Induced Developments
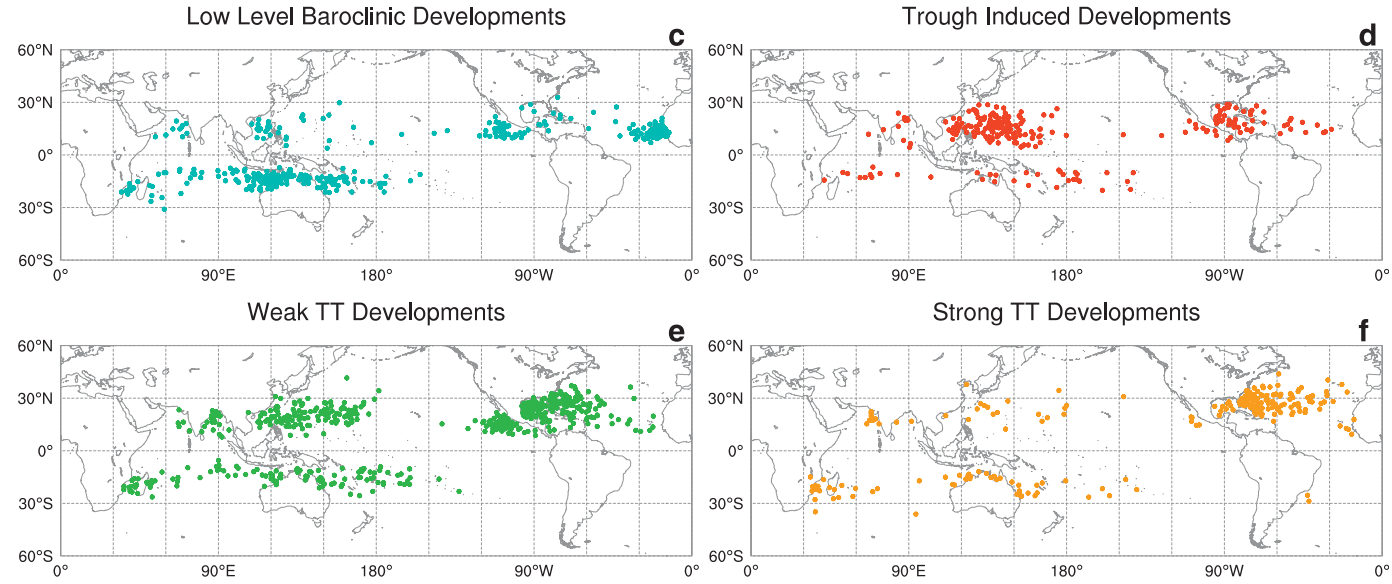

FIG. 7. (a)-(f) Development locations for all TCs in the climatology (circles) along specific development pathways as indicated. In (a), the color scheme follows that of the other panels in the figure: nonbaroclinic (blue), low-level baroclinic (cyan), trough induced (red), weak TT (green), and strong TT (orange). 

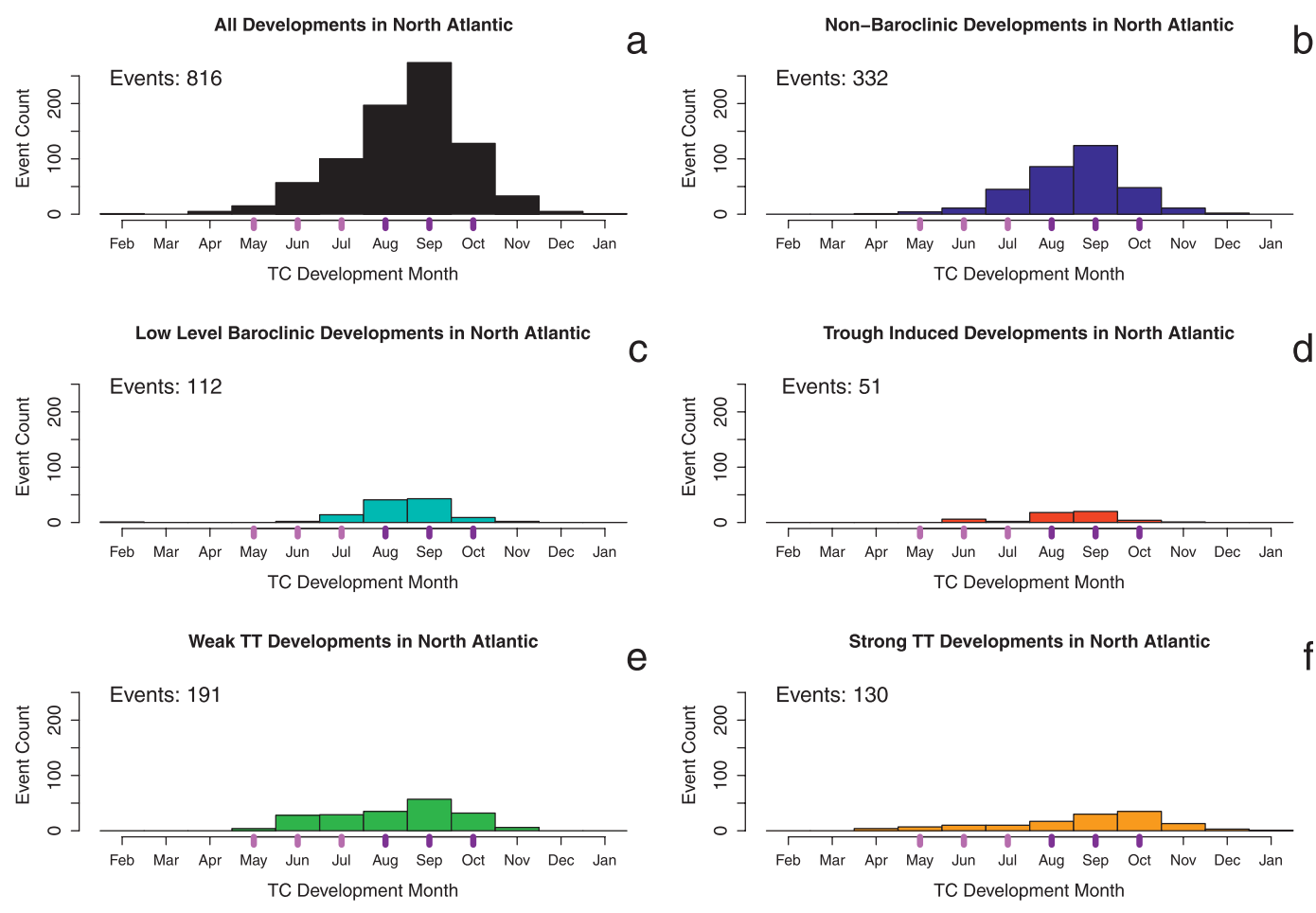

FIG. 8. Seasonal cycle of TC developments in the North Atlantic basin. (a) The full climatology and (b)-(f) pathway-specific cycles are shown as indicated in the panel titles. The $y$-axis range is held constant for all panels, and the total number of events in each class is indicated at the top left of each panel. The early- and late-summer seasons are identified with magenta and violet tick marks, respectively, for consistency with seasonality discussions in section 3. The hurricane season is centered by hemisphere for all similar plots to ease comparison, with the Northern Hemisphere plots beginning in February and the Southern Hemisphere plots beginning in August.

occur close to the Central American coast (Fig. 7c) and may thus have more direct socioeconomic impacts than storms that form farther west.

A clustering of low-level baroclinic developments appears downstream of each equatorial landmass (Africa, Central America, Australia) as shown in Fig. 7c. Mozer and Zehnder (1996), Molinari et al. (1997), and Serra et al. (2010) show that differential heating across the Central American coast and flow deflection by the Mexican plateau lead to the formation of a baroclinic easterly jet near $95^{\circ} \mathrm{W}$ that satisfies the conditions for combined barotropic and baroclinic instability (Charney and Stern 1962). The associated lower-tropospheric thermal gradients are consistent with TC development following the low-level baroclinic pathway (Serra et al. 2008; Blake and Pasch 2010). Easterly waves, either formed in situ or of Atlantic origin are frequently implicated in east Pacific tropical cyclogenesis (Avila 1991; Molinari et al. 1997; and others). Serra et al. (2008) find that the coherency of easterly waves in the eastern Pacific diminishes west of $120^{\circ} \mathrm{W}$, where background vertical shear and convective activity over warmer SSTs impact wave structure. These factors appear to explain the restriction of these events to the extreme eastern edge of the basin in the climatology.

Weak TT events are far less common in the east Pacific than in the western North Atlantic despite the proximity of the basins (cf. Figs. $8 \mathrm{e}$ and $9 \mathrm{e}$ ). The east Pacific lies outside the bounds of an early summer maximum in stratospheric PV streamer frequency on the subtropical tropopause (Wernli and Sprenger 2007, their Fig. 6d) consistent with the position of the North Atlantic TUTT (Fig. 4g). Instead, a fall maximum occurs just west of Baja California (their winter Fig. 4e, noted in the text to be similar to the fall $350-\mathrm{K}$ PV streamer frequency) in the climatological exit region of the North Pacific jet, possibly leading to the relative increase of late summer weak TT frequency in the basin (Bell and Bosart 1994).

The presence of an alongshore SST gradient between the cold California Current and the combined effect of land surface heating and a local warm countercurrent (Monk 1950) ensures the presence of moderate background baroclinicity (Fig. 1). Accordingly, trough incursions into the region may trigger weak TT rather than trough-induced or strong TT developments. 


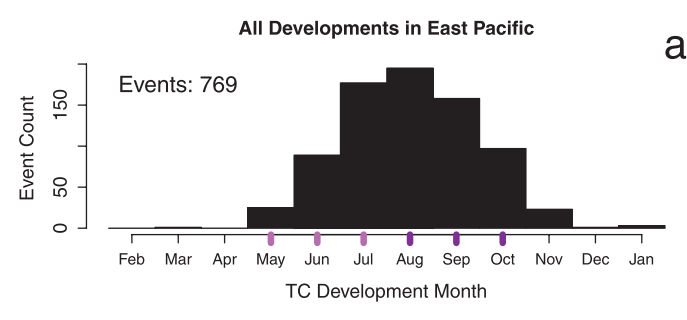

\section{a}
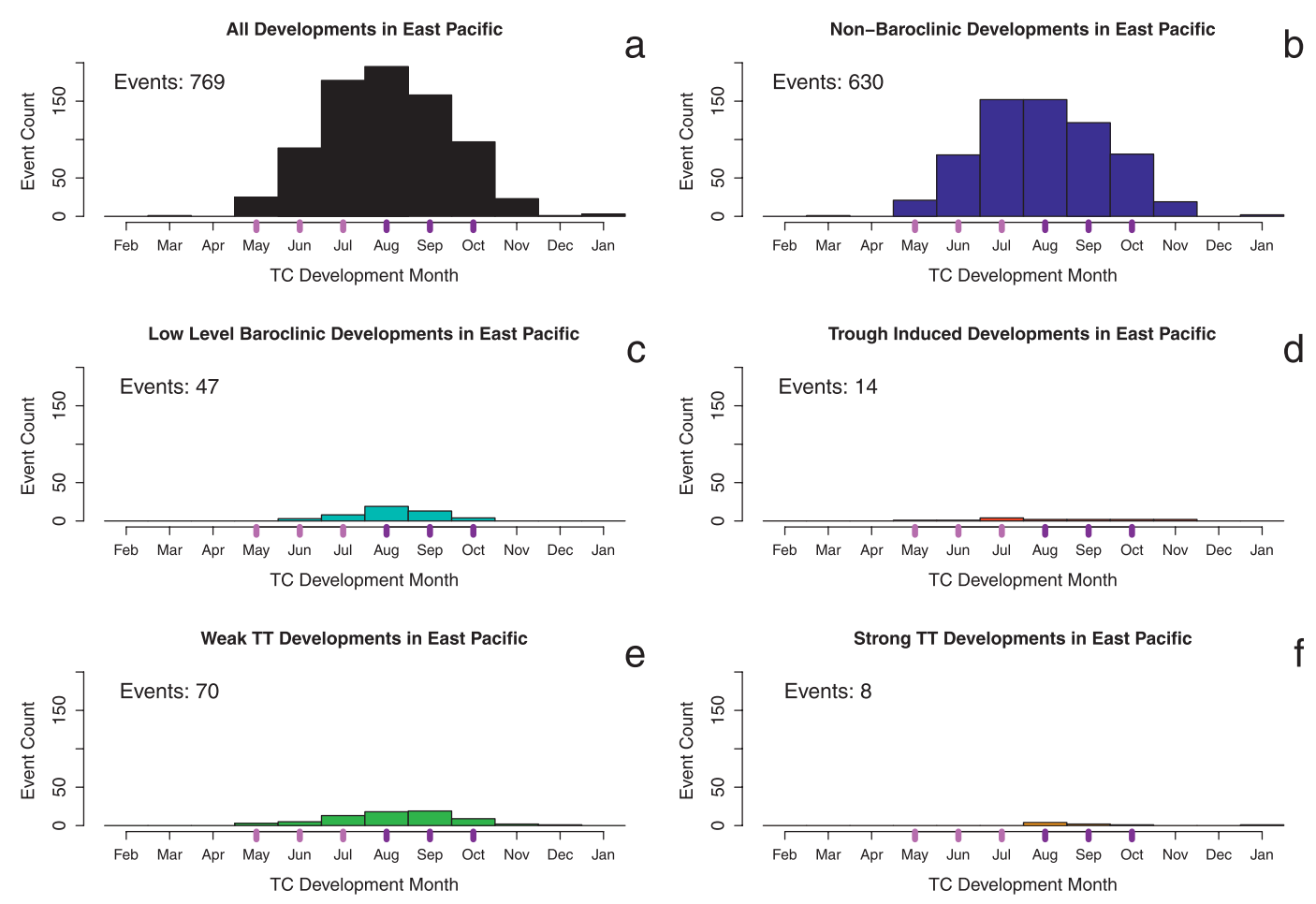

FIG. 9. Seasonal cycle of TC developments as in Fig. 8, but for the east Pacific basin.

\section{3) West PACIFIC BASIN}

The west Pacific basin is the most prolific in terms of TC count, with nonbaroclinic developments occurring in all months of the year (Figs. 6 and 10b). Ritchie and Holland (1999) present a comprehensive climatology of west Pacific tropical cyclogenesis, primarily from the perspective of nonbaroclinic, monsoon-influenced environments. Unlike any other region, the trough-induced pathway is the preferred secondary mode for TC development, with an average of 2.3 trough-induced developments per year, most of which occur in late summer (Fig. 10d). The relative frequency of both low-level baroclinic and strong TT developments reaches a global minimum in this basin, with these pathways accounting for less than $3 \%$ of developments (Fig. 6).

The importance of isolated cyclonic $\mathrm{PV}$ anomalies to TC development in the west Pacific is consistent with the high frequency of trough-induced events in the basin (Sadler 1967). A composite study of these features by Kelley and Mock (1982) demonstrates that ascent and cloudiness are concentrated in the southeastern quadrant of the disturbances, consistent with QG forcing given the easterly low-level flow relative to the upperlevel center. The spatial distribution of these events shows that development tends to occur near the end of the PV anomaly life cycle [cf. Fig. 7d here and Fig. 1 of
Kelley and Mock (1982)], as a result of enhanced deep convection in the developing circulation over the western Pacific warm pool that actively erodes the upperlevel disturbance (Sakamoto and Takahashi 2005).

The warm pool region that extends from the Philippines to approximately $160^{\circ} \mathrm{E}$ is unsupportive of baroclinicity equatorward of $20^{\circ} \mathrm{N}$. Differential surface fluxes have a strongly frontolytic effect on any baroclinic zone penetrating equatorward, reducing it rapidly to a shear line (Chen et al. 2007). Serra et al. (2008) show that the baroclinicity associated with easterly waves in the east Pacific basin has been eliminated by the time they reach the date line; thus, the direct contribution by easterly waves to TC development in the west Pacific found by Ritchie and Holland (1999) (18\%) and Chen et al. (2008) $(10 \%-25 \%)$ would be classified as nonbaroclinic in the current climatology. Without frontal or baroclinic wave features, development following either the lowlevel baroclinic or the strong TT pathway requires landsea thermal contrasts. This limits the areas affected by these development pathways to the South China Sea and the East China Sea along the Kuroshio (Figs. 1 and 7c,f).

The meridional gradient of SST increases between $20^{\circ}$ and $25^{\circ} \mathrm{N}$, slightly enhancing background baroclinicity compared to the warm pool region. The restricted weak TT season (Fig. 10e) coincides with the subtropical western North Pacific monsoon (Ueda and Yasunari 

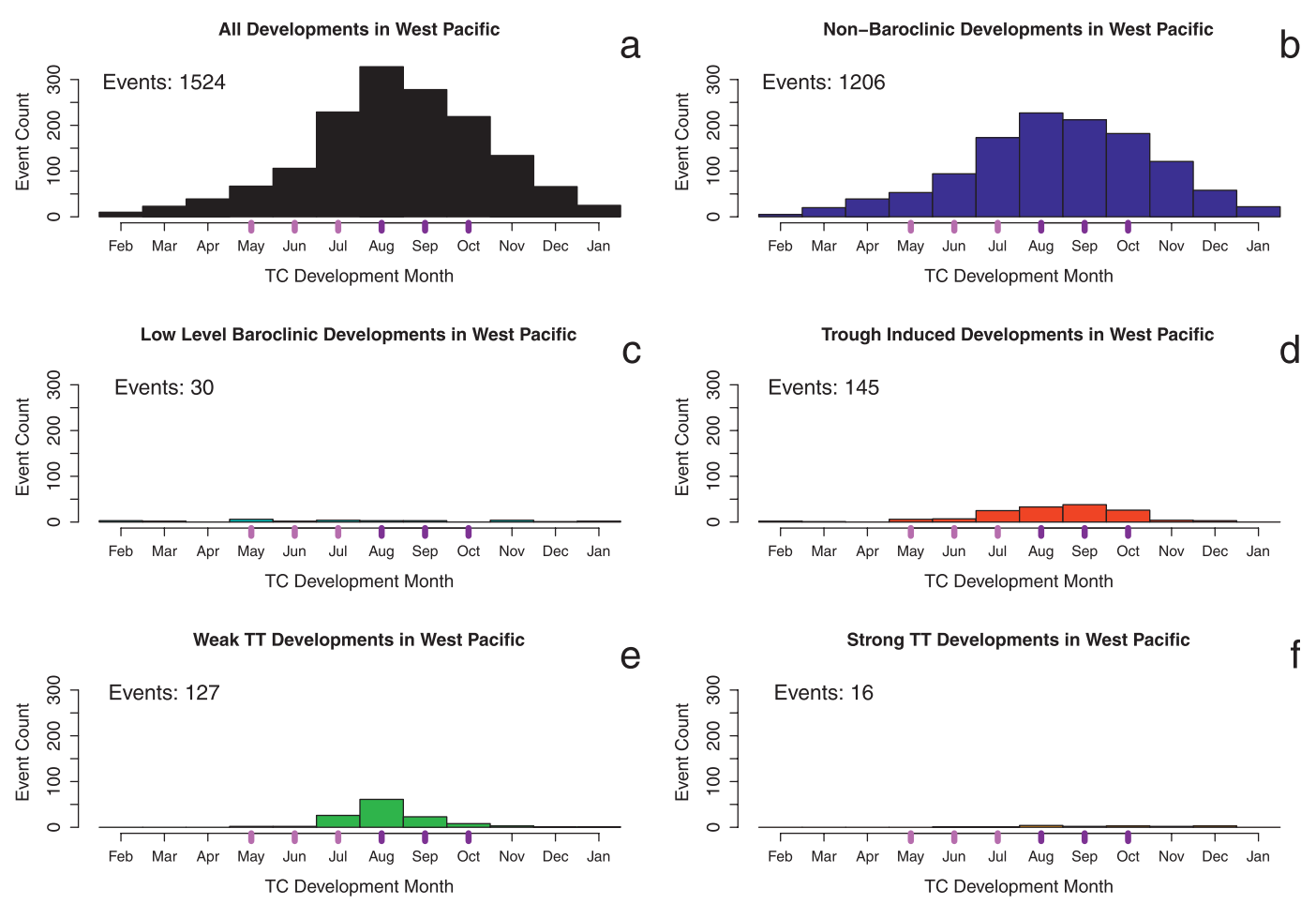

FIG. 10. Seasonal cycle of TC developments as in Fig. 8, but for the west Pacific basin.

1996), a pattern conducive to TC development whose establishment depends on the westward shift of the TUTT axis in late July (Sato et al. 2005; Lu et al. 2007). The associated subtropical PV streamer frequency reaches its maximum near the date line (Wernli and Sprenger 2007), consistent with the eastward shift of the weak TT development region compared to that of the trough-induced pathway (cf. Figs. 7d,e).

\section{4) NORTH INDIAN BASIN}

Despite having the fewest TCs of the global basins, formations in the north Indian Ocean follow a broad spectrum of development pathways as shown in Fig. 6 . The spatial (Fig. 7) and temporal distributions of development events are largely governed by the Indian summer monsoon, whose vertical shear and northward displacement of the monsoon trough suppress tropical cyclogenesis in the region except during the spring and fall transitional seasons (Gray 1968; Lee et al. 1989).
The Indian subcontinent naturally divides the region into two subbasins: the Arabian Sea west of $77^{\circ} \mathrm{E}$ and the Bay of Bengal east of this longitude (Evan and Camargo 2011). Each of these subbasins has a unique distribution of TC development pathways as shown in Table 5 and Figs. 11 and 12. The Bay of Bengal is the more prolific of the subbasins, and does not support formations involving lower-level thermal gradients. Conversely, Arabian Sea developments tend to occur in baroclinic environments, with low-level baroclinic and strong TT events accounting for $48 \%$ of formations in the region.

Early in the boreal summer, a strong cross-equatorial flow is established at low levels $(850 \mathrm{hPa})$ along the east coast of Africa (Findlater 1969, 1971). The Somali jet component of this circulation continues northeastward from the Horn of Africa into the Arabian Sea (Fig. 1 of Findlater 1971). The surface wind stress under the southwesterly jet core yields strong Ekman transport away from the coast and cold upwelling in the western

TABLE 5. Development pathway distributions in the north Indian subbasins.

\begin{tabular}{|c|c|c|c|c|c|c|c|c|c|c|}
\hline \multirow[b]{2}{*}{ Subbasin } & \multicolumn{2}{|c|}{ Nonbaroclinic } & \multicolumn{2}{|c|}{ Low-level baroclinic } & \multicolumn{2}{|c|}{ Trough induced } & \multicolumn{2}{|c|}{ Weak TT } & \multicolumn{2}{|c|}{ Strong TT } \\
\hline & Frequency & Count & Frequency & Count & Frequency & Count & Frequency & Count & Frequency & Count \\
\hline Bay of Bengal & $66 \%$ & 79 & $0 \%$ & 0 & $8 \%$ & 10 & $24 \%$ & 29 & $2 \%$ & 2 \\
\hline Arabian Sea & $35 \%$ & 14 & $28 \%$ & 11 & $5 \%$ & 2 & $12 \%$ & 5 & $20 \%$ & 8 \\
\hline
\end{tabular}



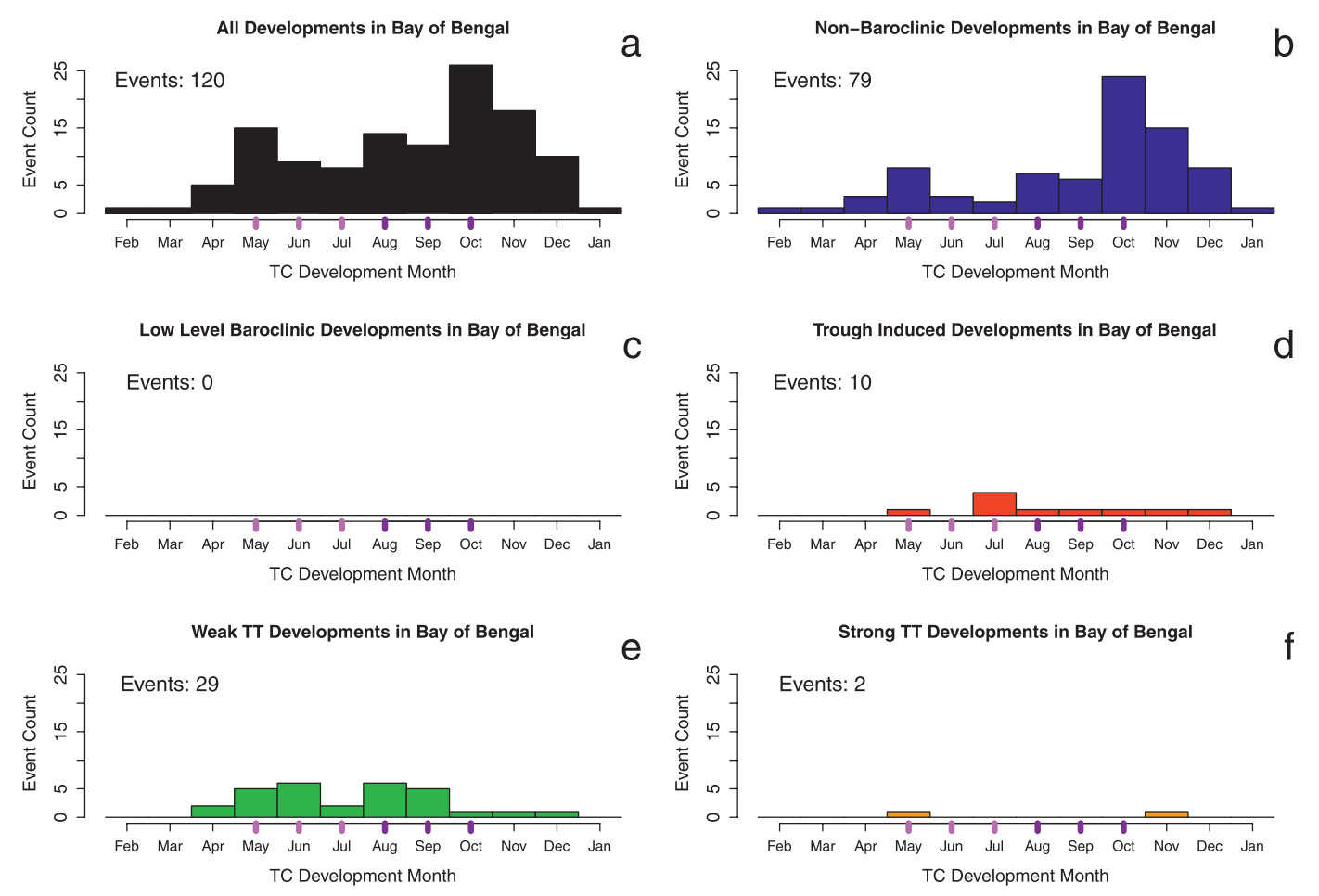

FIG. 11. Seasonal cycle of TC developments as in Fig. 8, but for the Bay of Bengal subbasin.

Arabian Sea (Düing and Leetmaa 1980). The importance of the resulting boundary layer baroclinicity to the structure and maintenance of the jet has been demonstrated by Krishnamurti and Wong (1979). No such upwelling occurs in the Bay of Bengal, leading to uniformly warm SSTs throughout the season (Shenoi et al. 2002).

The significant positive correlation between the strength of the Somali jet and TC development in the Arabian Sea is indicative of the early-summer importance of the low-level baroclinic pathway in the subbasin shown in Fig. 12 (Evan and Camargo 2011). Cyclonic shear on the poleward flank of the jet provides a favorable environment for tropical cyclogenesis, as does the increase in low-level moist static energy associated with strong surface enthalpy fluxes beneath the jet core (Boos and Emanuel 2009). However, strong monsoon-induced vertical shear between the westerly jet and the easterly flow aloft limits the number of storms that develop in the region (Evan and Camargo 2011).

The monsoon exhibits significant intraseasonal variability, particularly in form of "break" periods in which precipitation shifts from the subcontinent into the Himalayan foothills (Gadgil and Joseph 2003 and references therein). Ramsawamy (1962) shows that monsoon breaks can be triggered by amplifying Rossby waves on the subtropical jet. A localized weakening of the mean meridional PV gradient over the Persian Gulf identifies the region in which such disturbances can grow [Fig. 10a of Postel and Hitchman (1999)]. During the monsoon break, the prevailing easterly flow over northern India can be replaced by a westerly jet south of the Tibetan Plateau for several days. The resulting anticyclonic shear vorticity over the north Indian Basin is favorable for TC development (Lee et al. 1989). Such developments would be classified as trough-induced, weak TT, or strong TT depending on the lower-level baroclinicity associated with the developing vortex, which might itself be traditionally identified as a monsoon depression (Rao 1976). This development mechanism is consistent with the enhanced relative frequency of these pathways in the monsoon season [56\% (33/59) in June-September compared to $22 \%$ (22/101) over the remainder of the season]; however, further study is needed to quantify the impact of subtropical jet perturbations on monsoon breaks and associated TC development.

\section{5) SOUTH INDIAN BASIN}

With 950 classified TC developments, the south Indian Ocean (extending from the East African coast to $135^{\circ}$ E, Fig. 1) is second only to the west Pacific as the most prolific basin in this study. The majority of events follow the nonbaroclinic pathway; however, a global maximum of low-level baroclinic developments occurs 

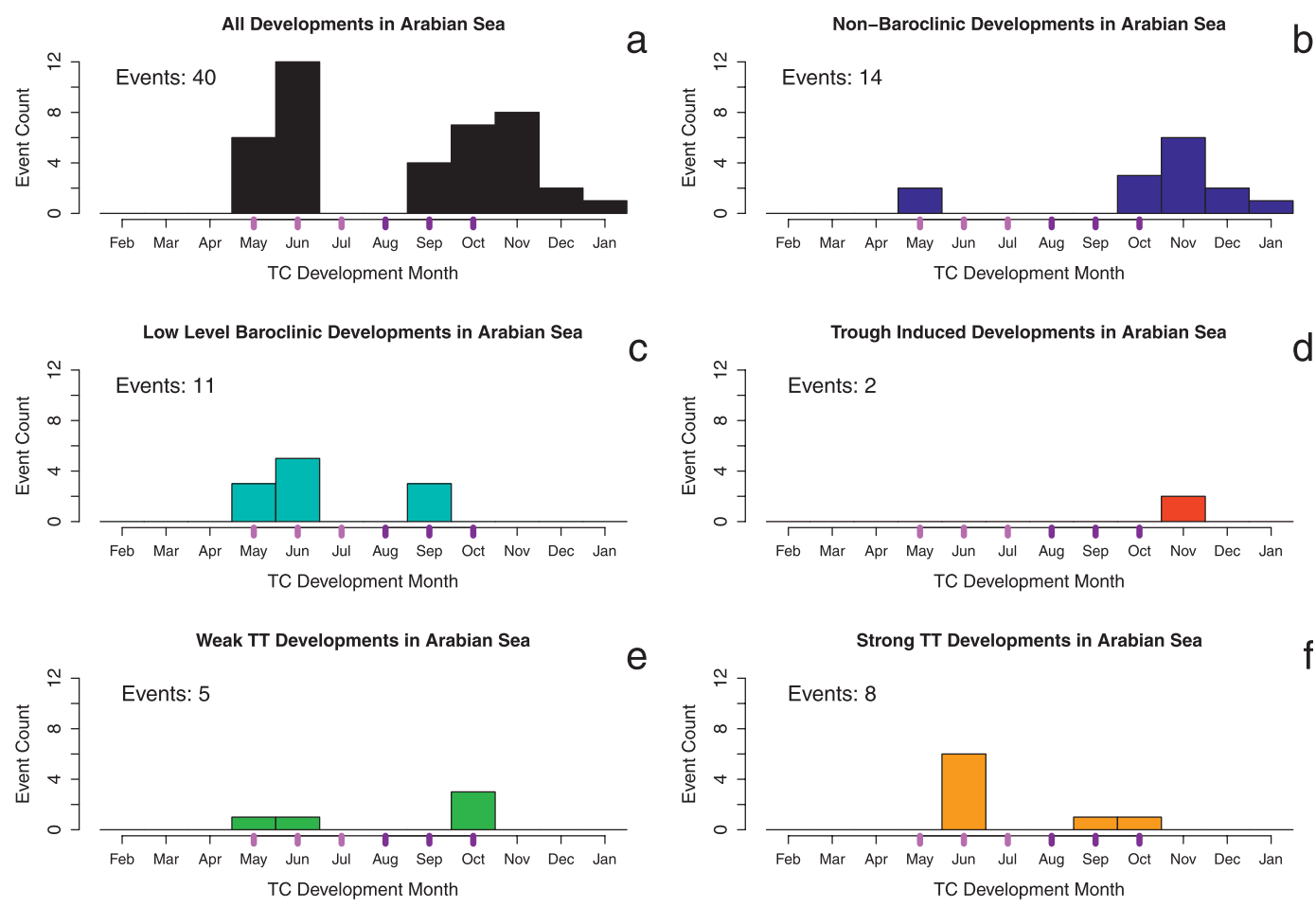

FIG. 12. Seasonal cycle of TC developments as in Fig. 8, but for the Arabian Sea subbasin.

in this region, which experiences an average of 2.3 such formations per year (Figs. 6 and 13c). The majority of these events occur between northwestern Australia and Indonesia, although some also take place in the Mozambique Channel and the south-central Indian Basin (Fig. 7c).

Throughout the peak of the Australian TC season (January-March), the monsoon trough lies along the northern periphery of the continent (McBride and Keenan 1982). A semipermanent heat low over northwestern Australia deforms the trough, creating a region of enhanced trade wind easterlies south of the trough axis. The increased lower-level cyclonic vorticity provides a favorable environment for TC development (Lee et al. 1989), and is necessarily associated with a thermal gradient established between the warm continental air of the heat low and the relatively cool air offshore [shown for TC Trixie (1975) in Fig. 18a of McBride and Keenan (1982)]. The fact that $49 \%$ of TCs in the Australian region form within $300 \mathrm{~km}$ of the continent (McBride and Keenan 1982) suggests that these temperature contrasts are important to development in the region, a finding that is consistent with the high density of low-level baroclinic formations shown in Fig. 7c.

Although the development of a TC following the weak TT or strong TT pathway near northwestern Australia is a relatively infrequent occurrence (Figs. 7e,f), McBride and Keenan (1982) emphasize the importance of upshear midlatitude troughs in $12 \%$ of the events that they investigate and note that Australian TC forecasters are trained to look for possible extratropical influences on pre-TC vortices in the region. As in the North Atlantic basin, the flattened seasonal distribution of these events implies that their relative frequency increases in the shoulder seasons (Figs. 13e,f) as the upper-level jet moves equatorward (M08).

In the western and central south Indian Basin, local maxima in the frequency of TC development following low-level baroclinic, weak TT, and strong TT pathways coexist (Fig. 7). Near the African coast, Nassor and Jury (1998) show that moist unstable boundary layers shift eastward during westerly wind bursts and create heavy rains over northern Madagascar. The lifting mechanism in these cases appears to be associated with tropicaltemperate troughs (Todd and Washington 1999), synopticscale baroclinic features of extratropical origin that strongly affect moisture convergence and convection. The development of TCs associated with these troughs in isolation would be categorized as low-level baroclinic; however, upper-level PV features also impact TC development in the region.

A local maximum in the frequency of Rossby wave breaks on the subtropical jet is shown by Postel and Hitchman (1999) and Ndarana and Waugh (2011) to 

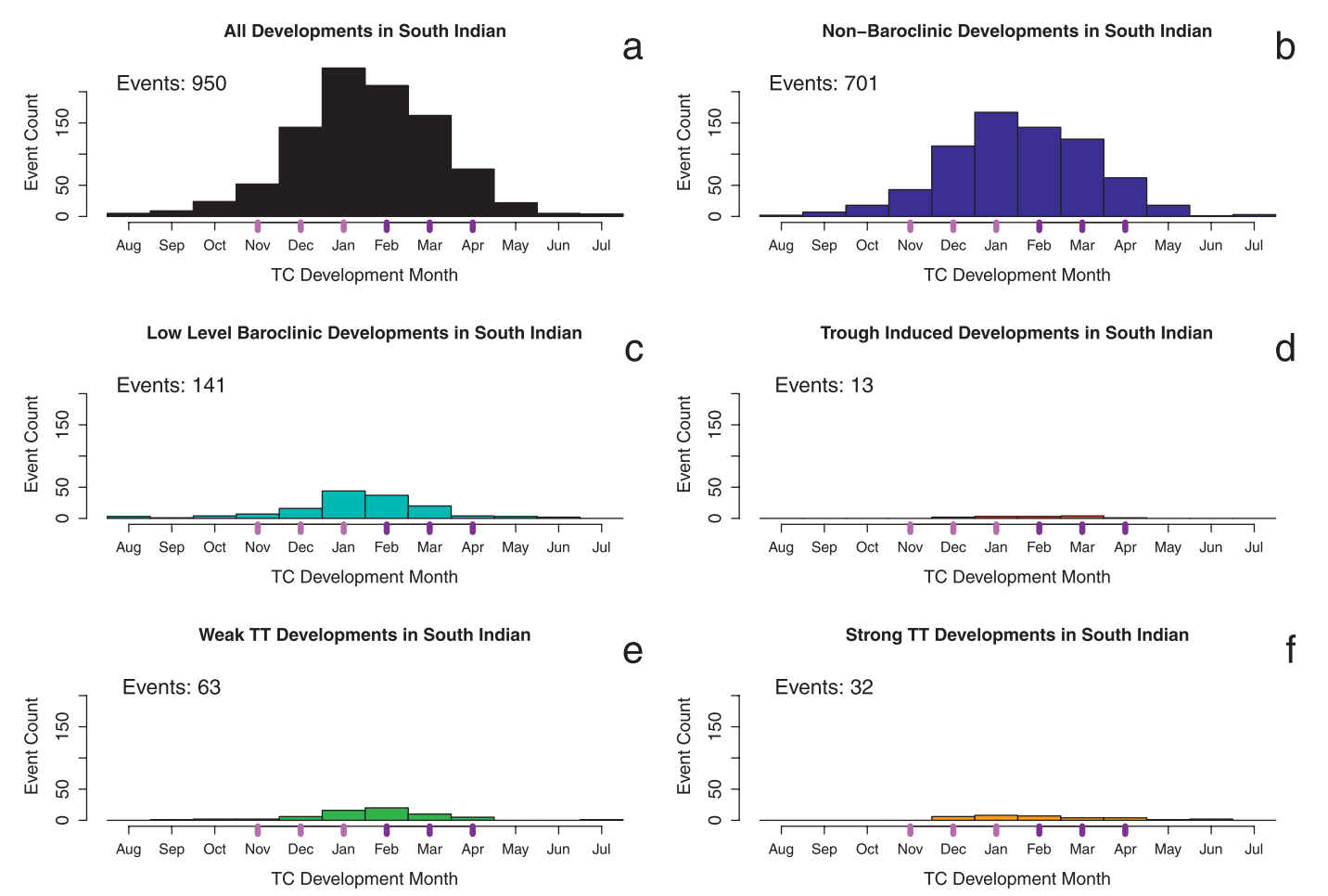

FIG. 13. Seasonal cycle of TC developments as in Fig. 8, but for the south Indian Basin.

occur over the central and western south Indian Ocean, where the incidence of trough-induced and weak TT developments peaks in the current climatology (Figs. 7d,e). Payne and Methven (2012) attribute PV-influenced TC developments in this region to the close proximity of the subtropical jet and the ITCZ. The poleward displacement of events close to the African coast, particularly notable for the weak TT and strong TT pathways (Figs. 7e,f), is likely a result of warm SSTs in the Agulhas Current [Gordon (1985) and Fig. 1]. The superposition of a western boundary current with a region of enhanced Rossby wave activity provides a background that is similar to that of the western North Atlantic region for TT-type developments, although both of the features are less pronounced in the south Indian Basin and TT events are accordingly less frequent.

\section{6) SOUTH PACIFIC BASIN}

The TC development pathway distribution in the South Pacific basin resembles that of the south Indian Ocean (Fig. 6), as does the seasonality of events (Fig. 14). The development mechanisms in the Australian region described in the previous subsection apply in the western portion of the basin; however, the Western Australian heat low is replaced by a secondary heat low feature centered over Queensland, Australia (Leslie 1980).
In addition to this quasi-permanent feature, McBride and Keenan (1982) describe how easterly surges south of the monsoon trough can enhance low-level cyclonic shear vorticity to aid the TC development process. Since these easterlies have their origins over the subtropical Pacific, thermal contrasts consistent with low-level baroclinic classification can be anticipated when the surges are sufficiently strong; however, the thermal structure of the flow is not diagnosed in the original study.

The East Australian Current (Hamon 1965) transports warm water poleward along the east coast of the continent, beneath a region with a weak local maximum in wave activity on the subtropical jet (Postel and Hitchman 1999; Ndarana and Waugh 2011). This establishes a favorable configuration for the trough-induced, weak TT, and strong TT pathways that rely on jet-level perturbations (Figs. 7d-f). The relative infrequency of these disturbances, however, limits the total number of formations following these pathways in the region.

Farther east, the South Pacific convergence zone (Trenberth 1976; Vincent 1994) separates northeasterly flow around the east Pacific subtropical high from southwesterly flow originating at higher latitudes east of Australia. This baroclinic feature is supported by both the eastward extension of the western Pacific warm pool and transient troughs of midlatitude origin (Kiladis et al. 1989). The South Pacific convergence zone creates an 

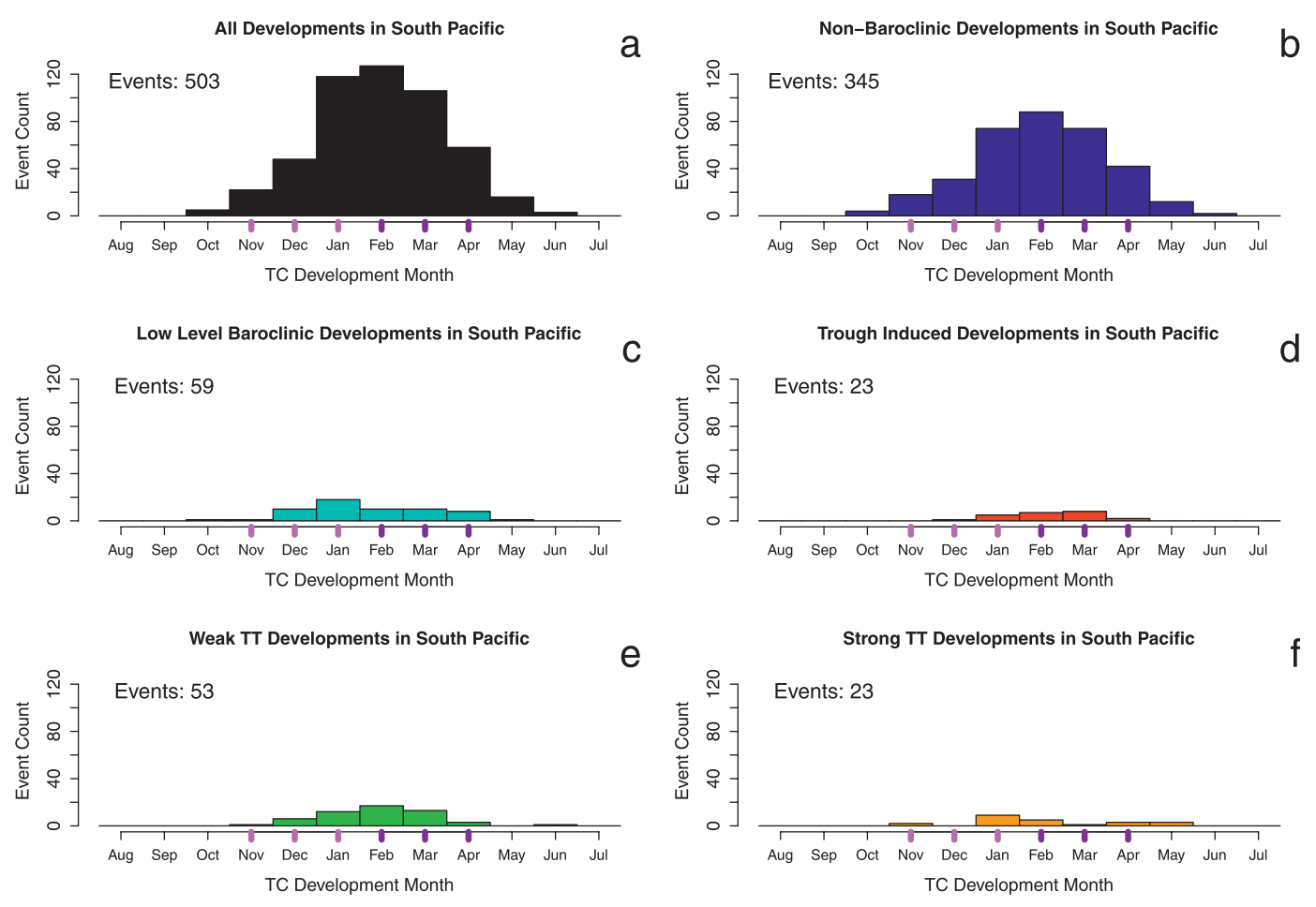

FIG. 14. Seasonal cycle of TC developments as in Fig. 8, but for the South Pacific basin.

environment conducive to TC development poleward of its northwest-southeast-tilted axis near the date line (Vincent et al. 2009). Consistent with its structure, weak TT events are almost twice as frequent near the convergence zone $\left[28 / 191,15 \% ; 170^{\circ} \mathrm{E}-135^{\circ} \mathrm{W}\right.$ following Vincent et al. (1991)] as they are in the rest of the basin $(25 / 312,8 \%)$. Further study is required to evaluate the influence of this "diagonal portion" of this feature on TC development pathways in the region.

\section{TC development efficiency}

With the exception of the nonbaroclinic pathway, the baroclinically influenced environments categorized in this climatology would generally be considered suboptimal for TC development (Lee et al. 1989). The results of the two previous sections are combined in order to assess the overall ability of different environments to generate TCs by investigating the pathway-specific ratio of development events to climatological frequency. This analysis does not imply that the pathway classifications should be considered a genesis potential index, since important parameters for cyclogenesis [including elevated SSTs, midlevel moisture, cyclonic vorticity, and weak vertical shear; Gray (1968)] are not directly accounted for in this study. However, in a climatological sense, pathways with detrimental characteristics will naturally be penalized in terms of their development to environmental frequency ratio (referred to hereafter as "efficiency" or "yield"). This quantity therefore indicates the frequency of TC formation in a given environment rather than the development potential of individual disturbances.

The global distribution of TC yield for each pathway is shown (see Fig. 16). The yield is computed using the ratio of the TC development density [Ramsay and Doswell III (2005); using a standard deviation of $10^{\circ}$, consistent with the spatial scale of metric calculations] to the number of days during which the pathway environment of interest was identified at each point: equivalent to dividing the appropriate panels of Fig. 7 by Fig. 4. Only points at which more than three development events following a particular pathway occurred over the course of the climatology are shaded in Fig. 15. The use of other radii in these calculations changes the structural detail and magnitude of the yield, but does not affect the following analysis.

The dominance of nonbaroclinic environments in the tropics (Figs. 4a,b) results in a nonbaroclinic yield (Figs. 15a,b) that corresponds closely to the TC development regions shown by Gray (1968). Seasonality in the yield is apparent in most basins, in part as a result of the late-summer SST increase that is implied by the poleward displacement of the $26.5^{\circ} \mathrm{C}$ isotherm in Fig. 15 . 


\section{Early Summer}
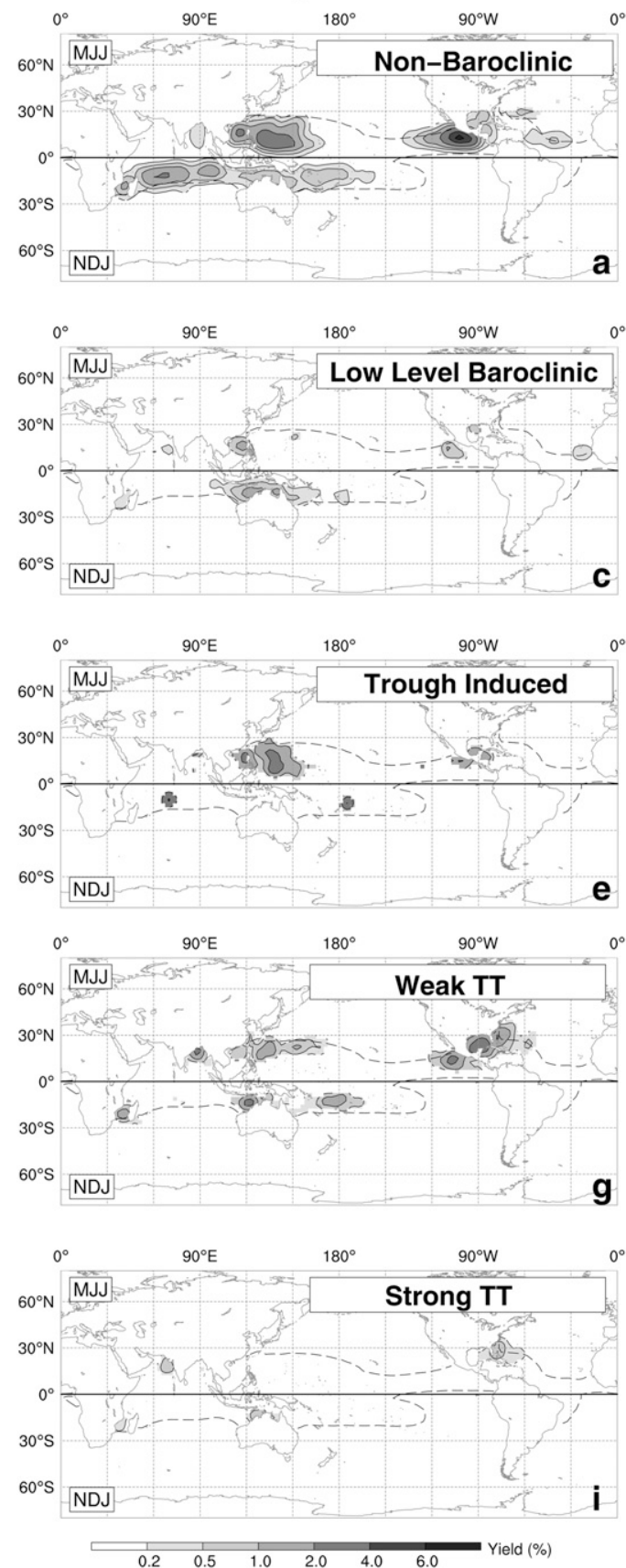

\section{Late Summer}
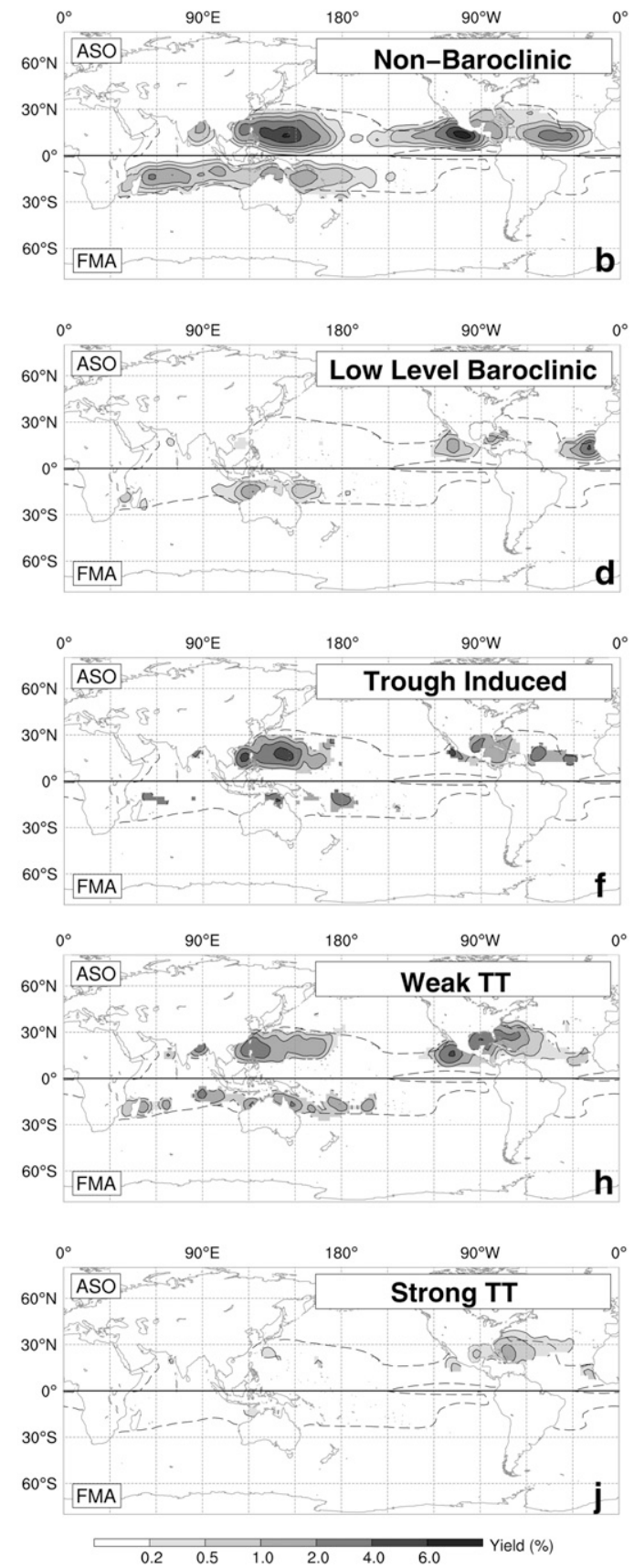

FIG. 15. Tropical cyclone development efficiency (in percentage as indicated on the grayscale bar) of pathways in the climatology for the (a),(c),(e),(g),(i) early summer and (b),(d),(f),(h),(j) late summer in each hemisphere. The layout of the panels follows that of Fig. 4, and the mean $26.5^{\circ} \mathrm{C} \mathrm{SST}$ isotherm (Reynolds et al. 2002) is plotted with a dashed line for reference (Gray 1968). The plotted efficiency levels correspond to the following per-event periods within $5^{\circ}$ of a given grid point: $0.2 \approx 1.5 \mathrm{yr} ; 0.5 \approx 6$ months; $1 \approx 3$ months; $2 \approx 2$ months; $4 \approx 1$ month; $6 \approx 2$ weeks.

Early season low-level baroclinic environments are relatively inefficient generators of TCs except near the northern Australian coastline (Fig. 15c). In late summer, yield values for low-level baroclinic formations peak globally in the eastern North Atlantic (Fig. 15d) in response to increased SSTs beneath the African easterly jet (Karyampudi and Pierce 2002). A very low yield (just over $0.2 \%$ ) is found for the Arabian Sea despite the link 
between TC development and jet- and upwellinginduced baroclinicity shown by Evan and Camargo (2011). This is likely an indication of the influence of additional external factors on TC formation in the region, including precursor vorticity and lower-level moisture.

Environments identified as favorable for the troughinduced pathway are second only to nonbaroclinic environments in terms of peak TC development efficiency, particularly in the active west Pacific basin (Figs. 15e,f). Although isolated cyclonic PV anomalies are relatively rare in the tropics (Figs. 4e,f), they account for a disproportionately large fraction of TC development events because of their elevated yield. Kelley and Mock (1982) describe how these features can enhance outflow channels and promote organized, sustained regions of ascent conducive to initial vortex intensification. Thereafter, the natural radiative weakening of the anomalies may be accelerated by vertical PV redistribution through deep convection, allowing the developing TC readily to overcome the effects of TUTT-induced vertical shear as it intensifies. The yields shown in Figs. 15e,f suggest that this is a remarkably efficient TC development mechanism.

Despite the prevalence of weak TT environments near the TUTT axes throughout the TC season (Figs. $4 \mathrm{~g}, \mathrm{~h}$ ), the weak TT pathway increases in efficiency only in late summer (Fig. 15h). In the south Indian Basin, infrequent late-season weak TT environments (Fig. 4h) are relatively effective TC generators as evidenced by the number of such formations between $80^{\circ}$ and $110^{\circ} \mathrm{E}$ shown in Fig. 7e. Payne and Methven (2012) attribute the late-season increase in south Indian baroclinic wave event-related TC developments to the proximity of the subtropical waveguide to the ITCZ. In the west Pacific basin, yields jump from $1 \%-5 \%$ between seasons, consistent with the late-June onset of the subtropical western North Pacific monsoon (Ueda and Yasunari 1996). The monsoon is characterized by increased lower-level convergence, cyclonic vorticity and convective activity (Wu et al. 2009), all of which favor TC development (Lee et al. 1989).

Across the Northern Hemisphere, climatological TUTT shear in the middle of the basins limits development and restricts high-yield weak TT regions to the peripheries of the basins (Figs. 15g,h). In the east and west Pacific, zonal minima in the frequency of weak TT environments (Figs. 4g,h) reduce the impact of this pathway on the total TC count. In the western North Atlantic basin, however, efficient late-summer weak TT environments occur frequently and locally increase the importance of the weak TT development pathway as described by Davis and Bosart (2002) and M08.

The generally low yield of the strong TT pathway evident in Figs. 15i,j is expected given the high vertical shears that are associated with this environment, particularly when the upshear trough has a large meridional scale (Hanley et al. 2001). The yield is maximized over the warmest SSTs in the North Atlantic basin, consistent with the need for strong destabilization and surface enthalpy fluxes to enhance convective momentum transports (Davis and Bosart 2002). Reduced upper-tropospheric temperatures in the trough permit TC formation over SSTs well below the $26.5^{\circ} \mathrm{C}$ limit proposed by Gray (1968), as evidenced by the extension of both weak TT and strong TT yields beyond this isotherm in Fig. 15. However, the low yield values in the central North Atlantic are indicative of the marginality of even these pathways under low-SST conditions (Emanuel 2005; McTaggart-Cowan et al. 2006).

The yields shown in Fig. 15 suggest that the underlying baroclinically influenced formation mechanisms are common across basins despite the dramatic interbasin variability in the relative importance of the different pathways shown in Fig. 6. For example, Fig. 15h shows that the peak ability of a TC to form in an environment identified as weak TT is roughly equivalent in the majority of the world's basins. The differences in relative formation frequency shown in Fig. 6 arise from the prevalence of the weak TT environment in regions conducive to TC formation rather than from fundamental differences in the TC development process. While such commonalities suggest that the climatology is discriminating between physically distinct subtypes of TC formation, any further analysis of development efficiency will require the integration of a genesis potential calculation into the diagnostic framework.

\section{Climatology in Q-Th metric space}

The discussions in the preceding sections have primarily been geographically based. This allows the pathway classifications to be related to features in the flow and for a basin-specific analysis of baroclinic influences on TC formation. However, further synthesis of the results can be achieved if each of these analyses is performed in $\mathbf{Q}-\mathrm{Th}$ metric space, wherein each data point is plotted in its appropriate position on the $\mathbf{Q}-\mathrm{Th}$ plane. (Technical information about this procedure can be found in the online supplement to this article.)

The nonbaroclinic state is found to dominate the environmental climatology (Fig. 16a), as expected from the geographical analysis. An axis of elevated climatological frequency (labeled "S" in Fig. 16a) extends into the strong TT environment, although the atmosphere still spends a relatively small amount of its time in this region of metric space. This appears to be a result of the high-latitude strong TT formations (Fig. 7f) that occur in 

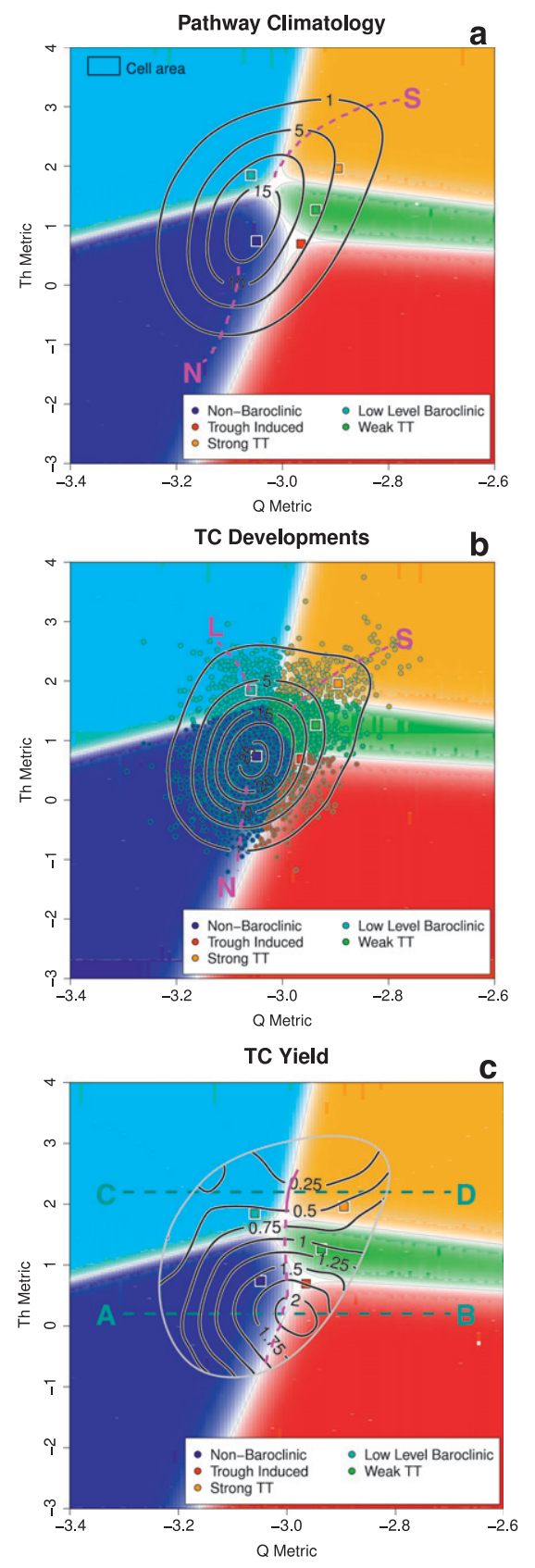

FIG. 16. (a) The environmental climatology in metric space for all points enclosed by the TC development density mask (shown in Fig. S1 of the online supplement) is shown in heavy black lines. Contouring represents the fraction of the time (\%) that the metrics lie close to a given position in metric space (the cell used to define proximity is shown in the top-left corner of the figure). (b) All cyclogenesis events classified in this study are plotted (dots color coded by development pathway, defined by their position on the plane), along with the fraction of TC developments that occur close to a given position in metric space [plotted with heavy black lines using the cell shown in (a)]. (c) The yield (heavy black lines) is shown for climatological frequencies $>1 \%$ (light gray solid line). Features described in the text are highlighted with dashed magenta lines and character annotations in each panel. The transition of the locations whose climatologies are dominated by baroclinic environments as shown in Figs. 4i,j (the applied mask is shown in the online supplement to this article).

The peak density of TC developments in the metric space is positioned near the centroid of the nonbaroclinic pathway (represented by a blue square in Fig. 16b), an anticipated result given the global dominance of this pathway (Fig. 6). Asymmetries in the kernel density estimate [a continuous function that represents the underlying distribution of TC developments across metric space (Duong 2007)] extend into both the strong TT and low-level baroclinic regions (labeled "S" and " $L$ " in Fig. $16 \mathrm{~b}$, respectively). Notable outliers also appear in those regimes, consistent with the broad spectrum of events that can occur when a vortex develops in the vicinity of a remnant frontal zone or in association with a breaking Rossby wave aloft (Davis and Bosart 2002; Hulme and Martin 2009a,b).

The rich structure of TC yield in metric space is revealed in Fig. 16c (computed as Fig. 16b divided by Fig. 16a). In this figure, the yield value of 1 represents an "average" efficiency, while relatively high- and lowyield regions of the metric space are represented by values greater and less than unity, respectively. A subtle shift of the feature labeled " $N$ " between the climatological and development frequencies (Figs. 16a,b) leads to an offset of the peak TC yield into the trough-induced region of metric space. This suggests that the pure nonbaroclinic environment does not provide the optimal state for TC development: the presence of an upper-level disturbance creates a more efficient TC-generating background (Gray 1988). As shown in Fig. 16c, a TC is $40 \%$ more likely to form in an environment with $\mathrm{Th}=0$ on the classification boundary between nonbaroclinic and trough induced, than it is at the nonbaroclinic centroid $(2.0 / 1.4=1.4)$.

Previous TC development climatologies have also reported on the prevalence of upper-level troughs during TC development (McBride and Keenan 1982; Briegel and Frank 1997; Bracken and Bosart 2000). In semiidealized studies, Challa and Pfeffer (1980) demonstrate the importance of eddy momentum flux convergence downshear of an upper-level trough, while Montgomery and Shapiro (1993) show that the diabatic coupling of

magenta line in (c) from dashed to solid corresponds to the change from the locus of maximum to minimum points for a given Th value. The background plotting follows the strategy employed in Fig. 2, and the planes of the yield cross section shown in Fig. 17 are plotted with dashed lines in (c). 
upper- and lower-level PV features is an important component of TC formation. Bracken and Bosart (2000) suggest that a delicate balance exists between beneficial QG forcing for ascent and the detrimental influence of associated deep-layer shear. This assertion is supported by the band of peak yield that lies near $\mathbf{Q}=-3$ in Fig. 16c (identified with a dashed magenta line). For a range of lower-level structures, this magnitude of upperlevel Q-vector convergence appears to represent the optimal balance between forcing and vertical shear. A cross section through the peak yield along $\mathrm{Th}=0.2$ (Fig. 17a) reveals a maximum near the classification boundary associated with an offset in the peaks of the climatological and development frequencies with increasing $\mathbf{Q}$.

The ridge of maximum yield near $\mathbf{Q}=-3$ for low $\mathrm{Th}$ values becomes a trough in yield values between the low-level baroclinic and strong TT classes (Fig. 16c). The decrease in yield with increasing Th implies that there are relatively few cases in which substantial lowerlevel thickness asymmetry is beneficial to TC development. However, lobes in the TC development density shown in Fig. 16b (features "S" and "L") suggest that favored development environments occur away from the boundary between the low-level baroclinic and strong TT classes.

This environmental preference is confirmed with a cross section (Fig. 17b) in which local yield maxima appear away from $\mathbf{Q} \sim-3$. Vortices forming in marginal environments characterized by strong lower-level baroclinicity thus appear to develop only if upper-level perturbations either are essentially absent, or are strong enough to play an integral role in the TT process. Precursors that interact with an upper-level feature of only moderate intensity may succumb to enhanced vertical shear without experiencing the benefits of forcing for ascent, possibly because the diabatic coupling suggested by Montgomery and Shapiro (1993) is more difficult to achieve when isentropic ascent is maximized well away from the center of the developing circulation. An analogous distinction is made between diabatic Rossby vortices and typical oceanic cyclones (Moore and Montgomery 2005): the structural and energetic characteristics of these features are fundamentally different because of the inability of PV anomalies to phase lock in intermediate cases. A rigorous determination of the physical processes that lead to the yield structures shown in Fig. 17c will require further study.

\section{Discussion}

Despite the dominance of nonbaroclinic TC developments across the majority of the tropical oceans ( $70 \%$ of all storms), this study has identified a rich spectrum of

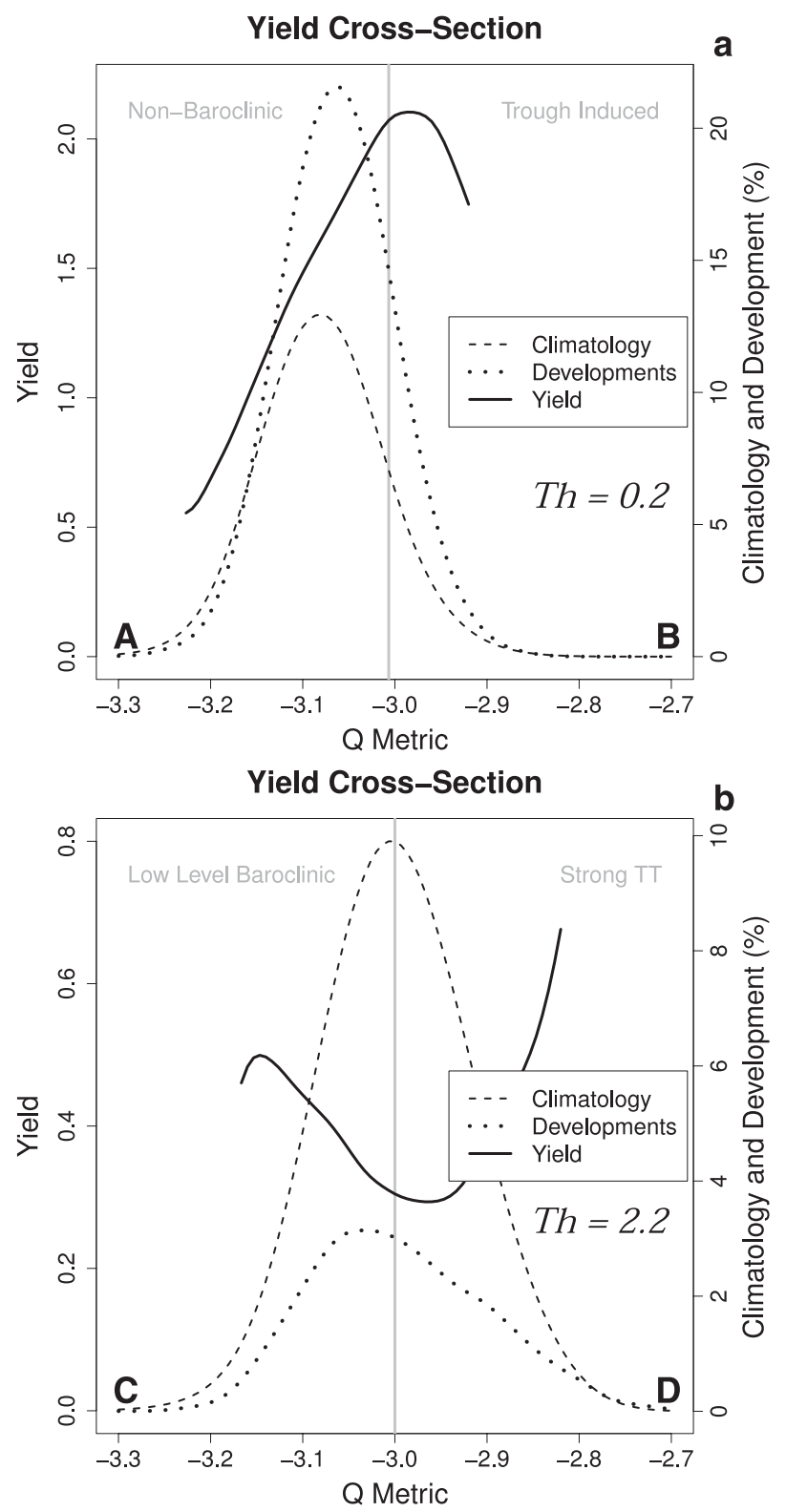

FIG. 17. Cross sections of TC yield along the (a) Th $=0.2$ and (b) $\mathrm{Th}=2.2$ contours as shown in Fig. $16 \mathrm{c}$ as $\mathrm{A}-\mathrm{B}$ and $\mathrm{C}-\mathrm{D}$, respectively. The yield (Fig. 16c) is plotted with a solid line using the primary $y$ axis, while the climatology (Fig. 16a) and development frequencies (Fig. 16b, in \%) are plotted with dashed and dotted lines against the alternate $y$ axis. The classification boundaries (both near $\mathbf{Q}=3$ ) are plotted with a gray line and the dominant categories are indicated at the top of the plot. The cross-sectional structures are insensitive to small changes in the chosen Th values.

formation events that occur in the presence of environmental baroclinicity. The low-level baroclinic pathway is followed by $8 \%$ of TCs, primarily in association with SST gradients and lower-level jets. The troughinduced environment is generally associated with a TUTT 
cell, although this relatively efficient pathway is infrequently encountered outside the west Pacific basin ( $6 \%$ of events globally). The strongest environmental baroclinicity is involved in weak TT and strong TT formations, pathways that account for $11 \%$ and $5 \%$, respectively, of TC developments worldwide.

The influence of TC development pathway on predictability is unknown, as is the ability of climate models to reproduce the climatological characteristics identified in this study. An investigation of these issues may yield improvements or insights into predictive skill over a range of time scales. In particular, the environmental pathway classification could be combined with traditional genesis potential evaluation techniques to develop a pathway-conditional index. Such a predictor would be better suited for a broad range of TC developments than the current forms that naturally focus on nonbaroclinic events.

The objective TC development pathway classification procedure developed here has allowed for a determination of the relative importance of each category to basin-total TC counts. It is hoped that this climatology will serve as a baseline for future composite and process studies studies that enhance our understanding of the role of baroclinic influences on TC formation both in specific regions and around the world.

Acknowledgments. Reanalysis-I data were obtained through NOAA/ESRL. Data from the ERA-40 and ERA-Interim were downloaded via the ECMWF data server, and JRA-25 data were obtained from the JMA data server. IBTrACS data were made available by NOAA/NCDC through the World Data Center for Meteorology, while NOAA OI SST(V2) fields were obtained from NOAA/OAR/ESRL PSD. Notable advancements in this work occurred during the lead author's extended visit to NCAR in April 2012 (funded by the MMM division). The authors also benefited from discussions with Chris Davis, Ayrton Zadra, John Gyakum, and Eyad Atallah during the development of the climatology and preparation of this manuscript. This work was supported in part by NSF Grants ATM-0849491 and AGS-0849356.

\section{APPENDIX}

\section{Details of the Classification Technique}

Linear discriminant analysis (Friedman 1989) is a statistical technique that uses a training dataset to "learn" how to classify categorical events based on a limited number of predictors (in this case two: $\mathbf{Q}$ and Th). Unlike the LTM method employed by M08, the LDA
TABLE A1. Synthesis chart for recombination of LTM-derived categories to final tropical cyclogenesis categories for the North Atlantic as in Table 2 of M08, but including the elimination of the transient trough interaction class. Metric group numbers refer to the trajectory identifications in Figs. 6 and 7 of M08.

\begin{tabular}{lccc}
\hline \hline \multicolumn{1}{c}{ Category } & $\begin{array}{c}\text { Membership } \\
\text { percentage }\end{array}$ & Q group & Th group \\
\hline Nonbaroclinic & 40 & 1 & 1 \\
& & 2 & 1 \\
& & 5 & 1 \\
& & 1 & 2 \\
Low-level baroclinic & \multirow{2}{*}{13} & 2 & 2 \\
& & 5 & 2 \\
Trough induced & & 1 & 3 \\
& & 2 & 3 \\
& & 5 & 3 \\
Weak TT & & 3 & 1 \\
& & 4 & 1 \\
& & 6 & 1 \\
Strong TT & & 7 & 1 \\
& & 3 & 2 \\
& & 4 & 2 \\
& & 6 & 2 \\
& & 7 & 2 \\
& & 3 & 3 \\
& & 4 & 3 \\
& & 6 & 3 \\
& & 7 & 3 \\
\hline
\end{tabular}

considers the metrics at a single analysis time only $\left(T_{0-12 \mathrm{~h}}\right)$. This is sufficient to discriminate between five of the six original classes because of the shallow slopes of the metric trajectories shown in Figs. 6 and 8 of M08. The relatively steep slope of the $\mathbf{Q}$ metric of the transient trough interaction category of M08 necessitates its elimination for this study. The Th metric is used to resynthesize these events into the trough-induced, weak TT, and strong TT classes as shown in Table A1.

Given these metric values and the resynthesized classifications (Table A1), the LDA performs a pair of linear transformations that seek to minimize withingroup variance while maximizing between-group variance. The LDA differs from cluster analysis in that the groupings are already known for the training dataset. Instead of identifying heterogeneity in metric space, the LDA manipulates the dimensions of the phase space to maximize group separations.

The basic steps of the LDA are straightforward. First, the prior probability of membership in class $k$ (one of the $K=5$ classes identified in Table A1) is estimated $\left(\pi_{k}\right)$. In this study, the proportional priors from the $N$-member LTM climatology are used,

$$
\pi_{k}=\frac{\text { count of TCs in class } k}{N},
$$


since it is clear from M08 that some TC development pathways are preferred over others. The results shown here are insensitive to small changes in the prescription of the priors $(10 \%-20 \%)$. Second, for a given set of metric values $x$, the posterior probability of membership in group $G$ for each class $k$ is computed as

$$
\operatorname{Pr}(G=k \mid x)=\frac{f_{k}(x) \pi_{k}}{\sum_{n=1}^{K} f_{n}(x) \pi_{n}},
$$

where the denominator represents a normalization factor that ensures that $\sum_{k=1}^{K} \operatorname{Pr}(G=k \mid x)=1$ and $f_{k}(x)$ is the probability density function (PDF) for class $k$, described in more detail below. Third, a discrete classification estimate is made by selecting the class with the maximum posterior probability:

$$
G(x)=\underset{k}{\arg \max } \operatorname{Pr}(G=k \mid x) .
$$

In addition to providing this "most likely" classification, the posterior of the LDA [Eq. (A2)] provides probabilistic information about the certainty of the class assignment. For example, if the metrics for a TC development event fall near the boundary between the nonbaroclinic and low-level baroclinic classes, then the posteriors may be roughly 0.5 for each of these categories.

The PDF for each class $k$, also called the class density estimate, is represented by a multivariate Gaussian function in LDA:

$$
f_{k}(\mathbf{x})=\frac{1}{(2 \pi)^{D / 2}|\mathcal{Q}|^{1 / 2}} e^{-1 / 2\left[\left(\mathbf{x}-\overline{\mathbf{x}}_{k}\right)^{\mathrm{T}} \mathcal{Q}\left(\mathbf{x}-\overline{\mathbf{x}}_{k}\right)\right]} .
$$

The centroid of class $k$ is the mean metric vector $\overline{\mathbf{x}}_{k}$, and $D=2$ represents the number of dimensions. The quantity $\mathcal{Q}$ in Eq. (A4) represents the estimate of the covariance matrix, taken to be identical for all classes. It is this assumption that separates the LDA from quadratic discriminant analysis and results in a set of identical Gaussian PDFs that are centered on the class means across the metric space.

There are two important benefits to using the combination of reanalysis datasets described in section $2 \mathrm{~b}$. The first is that the use of the ensemble-mean metrics should, on average, provide a higher quality estimate of the true metric value (Leith 1974). The second is that the spread between the individually-computed reanalysis metrics is indicative of the uncertainty of the analyzed state to the extent that a spread-skill relationship holds. The latter is particularly important because it allows for the conflation of classification uncertainty (represented
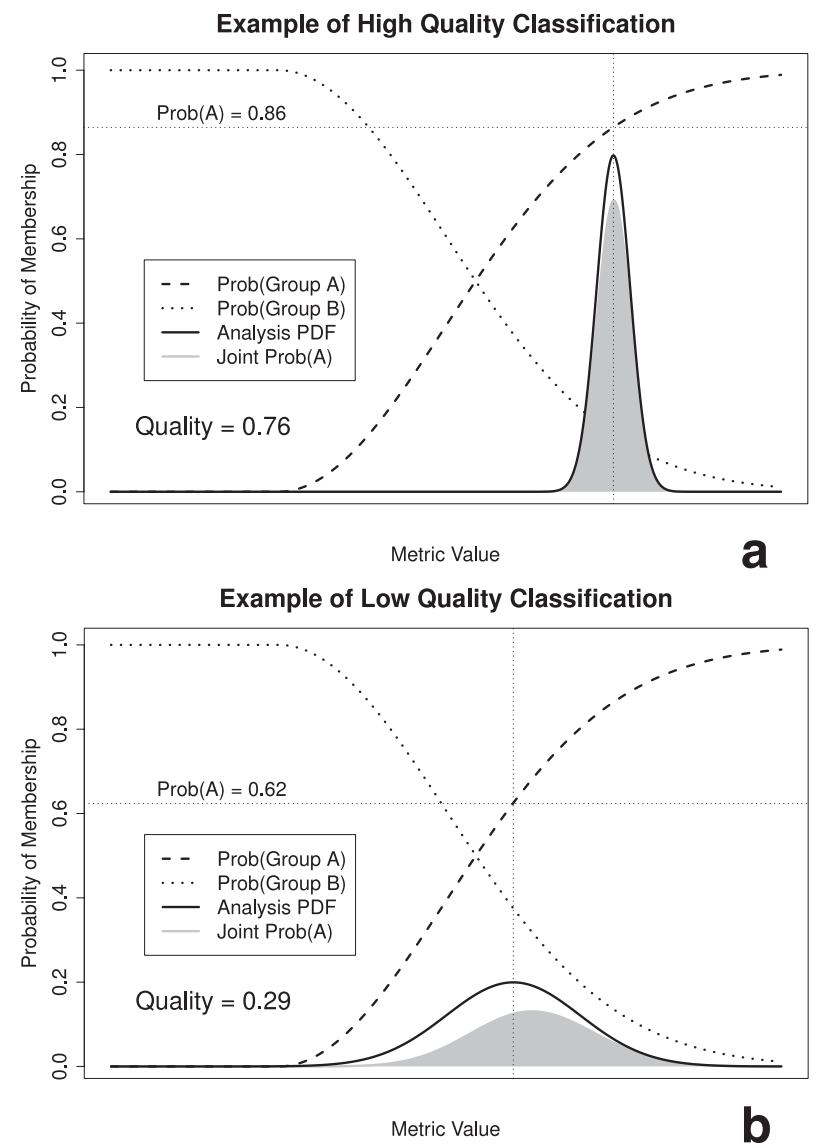

FIG. A1. Schematic representation of quality assessment in one dimension for two classes. The posterior probabilities [denoted "Prob( )" in the legend] of membership in classes A and B are shown with dashed and dotted lines, respectively. (a) A highquality value $(0.76)$ is assigned because the narrow analysis PDF (solid line) and mean metric placement results in a high joint probability of membership in class A (gray shading). (b) A lowquality value (0.29) is assigned because of large spread in the analysis PDF whose mean value lies near the classification boundary.

by the posteriors) and analysis uncertainty (represented by the variance of the reanalysis metrics, with mean climatological values of 0.25 and $6.4 \times 10^{-3}$ for the Th and $\mathbf{Q}$ metrics, respectively, when less than three reanalyses are available for a given event: the results are insensitive to small changes in these values) into an overall "quality" assessment that can be used to estimate the reliability of a specific classification. This is accomplished by creating a joint PDF from the class density estimate [Eq. (5)] of the discrete classification and a multivariate Gaussian function of the same form but with $\overline{\mathbf{x}}_{k}$ and $\mathbf{Q}$ representing the ensemble mean and variance. The quality value assigned to classification is the integral of this joint PDF, normalized to lie in $[0,1]$.

This is conceptually equivalent to placing Gaussian humps at each TC development location in Fig. 2 rather 
than treating each event's position on the phase space as a single point, and is shown schematically for two classes in one metric dimension in Fig. A1. In Fig. A1a, small analysis uncertainty is combined with a high posterior probability (0.86) to yield a high-quality classification (0.76). Conversely, Fig. A1b shows a case in which large analysis uncertainty and proximity to a category boundary (posterior probability of 0.62 ) result in low estimated quality for the classification (0.29). Studies that are sensitive to misclassifications will be able to make use of this additional information to create subsets of highly reliable events as shown in Fig. 5.

\section{REFERENCES}

Appenzeller, C., and H. C. Davies, 1992: Structure of stratospheric intrusions into the troposphere. Nature, 358, 570-572.

Avila, L. A., 1991: Eastern North Pacific hurricane season of 1990. Mon. Wea. Rev., 119, 2034-2046.

Belkin, I. M., and A. L. Gordon, 1996: Southern ocean fronts from the Greenwich meridian to Tasmania. J. Geophys. Res., 101, 3675-3696.

Bell, G. D., and L. F. Bosart, 1994: Midtropospheric closed cyclone formation over the southwestern United States, the eastern United States, and the Alps. Mon. Wea. Rev., 122, 791-813.

Blake, E. S., and R. J. Pasch, 2010: Eastern North Pacific hurricane season of 2008. Mon. Wea. Rev., 138, 705-721.

Boos, W. R., and K. A. Emanuel, 2009: Annual intensification of the Somali jet in a quasi-equilibrium framework: Observational composites. Quart. J. Roy. Meteor. Soc., 135, 319-335.

Bosart, L. F., and J. A. Bartlo, 1991: Tropical storm formation in a baroclinic environment. Mon. Wea. Rev., 119, 1979-2013.

Bracken, W. E., and L. F. Bosart, 2000: The role of synoptic-scale flow during tropical cyclogenesis over the North Atlantic Ocean. Mon. Wea. Rev., 128, 353-376.

Braun, S. A., 2010: Reevaluating the role of the Saharan air layer in Atlantic tropical cyclogenesis and evolution. Mon. Wea. Rev., 138, 2007-2037.

Briegel, L. M., and W. M. Frank, 1997: Large-scale influences on tropical cyclogenesis in the western North Pacific. Mon. Wea. Rev., 125, 1397-1413.

Burpee, R. W., 1972: The origin and structure of easterly waves in the lower troposphere of North Africa. J. Atmos. Sci., 29, 77-90.

Challa, M., and R. L. Pfeffer, 1980: Effects of eddy fluxes of angular momentum on model hurricane development. J. Atmos. Sci., 37, 1603-1618.

Charney, J. G., and M. E. Stern, 1962: On the stability of internal baroclinic jets in a rotating atmosphere. J. Atmos. Sci., 19, 159172.

Chen, G. T.-J., and L.-F. Chou, 1994: An investigation of cold vortices in the upper troposphere over the western North Pacific during the warm season. Mon. Wea. Rev., 122, 14361448.

_ C.-C. Wang, and A.-H. Wang, 2007: A case study of subtropical frontogenesis during a blocking event. Mon. Wea. Rev., 135, 2588-2609.

Chen, T.-C., S.-Y. Wang, M.-C. Yen, and A. J. Clark, 2008: Are tropical cyclones less effectively formed by easterly waves in the western North Pacific than in the North Atlantic? Mon. Wea. Rev., 136, 4527-4540.
Colton, D. E., 1973: Barotropic scale interactions in the tropical upper troposphere during the northern summer. J. Atmos. Sci., 30, $1287-1302$.

Davis, C., and L. F. Bosart, 2002: Numerical simulations of the genesis of Hurricane Diana (1984). Part II: Sensitivity of track and intensity prediction. Mon. Wea. Rev., 130, 1100-1124.

— and - 2003: Baroclinically induced tropical cyclogenesis. Mon. Wea. Rev., 131, 2730-2747.

— transition of cyclones. Bull. Amer. Meteor. Soc., 85, 1657-1662.

Dee, D. P., and Coauthors, 2011: The ERA-Interim Reanalysis: Configuration and performance of the data assimilation system. Quart. J. Roy. Meteor. Soc., 137, 553-597.

Düing, W., and A. Leetmaa, 1980: Arabian Sea cooling: A preliminary heat budget. J. Phys. Oceanogr., 10, 307-312.

Dunion, J. P., and C. S. Velden, 2004: The impact of the Saharan air layer on Atlantic tropical cyclone activity. Bull. Amer. Meteor. Soc., 85, 353-365.

Duong, T., 2007: ks: Kernel density estimation and kernel discriminant analysis for multivariate data in R. J. Stat. Software, 21, 1-16.

Elsner, J. B., G. S. Lehmiller, and T. B. Kimberlain, 1996: Objective classification of Atlantic hurricanes. J. Climate, 9, 2880-2889.

Emanuel, K. A., 2005: Genesis and maintenance of "Mediterranean hurricanes." Adv. Geosci., 2, 1-4.

Erickson, C. O., 1971: Diagnostic study of a tropical disturbance. Mon. Wea. Rev., 99, 67-78.

Evan, A. T., and S. J. Camargo, 2011: A climatology of Arabian Sea cyclonic storms. J. Climate, 24, 140-158.

Evans, J. L., and M. P. Guishard, 2009: Atlantic subtropical storms. Part I: Diagnostic criteria and composite analysis. Mon. Wea. Rev., 137, 2065-2080.

Ferreira, R. N., and W. H. Schubert, 1999: The role of tropical cyclones in the formation of tropical upper-tropospheric troughs. J. Atmos. Sci., 56, 2891-2907.

Findlater, J., 1969: A major low-level air current near the Indian Ocean during the northern summer. Quart. J. Roy. Meteor. Soc., 95, 362-380.

_ 1971: Mean monthly airflow at low levels over the western Indian Ocean. Geophys. Mem., 16, 1-53.

Fitzpatrick, P. J., J. A. Knaff, C. W. Landsea, and S. V. Finley, 1995: Documentation of a systematic bias in the aviation model's forecast of the Atlantic tropical upper-tropospheric trough: Implications for tropical cyclone forecasting. Wea. Forecasting, 10, 433-446.

Friedman, J. H., 1989: Regularized discriminant analysis. J. Amer. Stat. Assoc., 84, 165-175.

Gadgil, S., and P. V. Joseph, 2003: On breaks of the Indian monsoon. J. Earth Syst. Sci., 112, 529-558.

Godfrey, J. S., 1996: The effect of the Indonesian throughflow on ocean circulation and heat exchange with the atmosphere: A review. J. Geophys. Res., 101 (C5), 12 217-12 237.

Goldenberg, S. B., and L. J. Shapiro, 1996: Physical mechanisms for the association of El Niño and west African rainfall with Atlantic major hurricane activity. J. Climate, 9, 1169-1187.

Gordon, A. L., 1985: Indian-Atlantic transfer of thermocline water at the Agulhas retroflection. Science, 227, 1030-1033.

Gray, W. M., 1968: Global view of the origin of tropical disturbances and storms. Mon. Wea. Rev., 96, 669-700.

_, 1988: Environmental influences on tropical cyclones. Aust. Meteor. Mag., 36, 127-139.

Guishard, M. P., J. L. Evans, and R. E. Hart, 2009: Atlantic subtropical storms. Part II: Climatology. J. Climate, 22, 3574-3594. 
Hamon, B. V., 1965: The East Australian current, 1960-1964. Deep-Sea Res. Oceanogr. Abstr., 12, 889-921.

Hanley, D. E., J. Molinari, and D. Keyser, 2001: A composite study of the interactions between tropical cyclones and uppertropospheric troughs. Mon. Wea. Rev., 129, 2570-2584.

Hess, J. C., J. B. Elsner, and N. E. LaSeur, 1995: Improving seasonal hurricane predictions for the Atlantic basin. Wea. Forecasting, 10, 425-432.

Hoskins, B. J., I. Draghici, and H. C. Davies, 1978: A new look at the $\omega$-equation. Quart. J. Roy. Meteor. Soc., 104, 31-38.

— M. McIntyre, and A. Robertson, 1985: On the use and significance of isentropic potential vorticity maps. Quart. J. Roy. Meteor. Soc., 111, 877-946.

Hulme, A. L., and J. E. Martin, 2009a: Synoptic and frontal scale influences on tropical transition events in the Atlantic basin. Part I: A six case survey. Mon. Wea. Rev., 137, 3626-3650.

$\longrightarrow$, and _ 2009b: Synoptic and frontal scale influences on tropical transition events in the Atlantic basin. Part II: Tropical transition of Hurricane Karen. Mon. Wea. Rev., 137, 3626-3650.

Kalnay, E., and Coauthors, 1996: The NCEP/NCAR 40-Year Reanalysis Project. Bull. Amer. Meteor. Soc., 77, 437-472.

Karyampudi, V. M., and T. N. Carlson, 1988: Analysis and numerical simulations of the Saharan air layer and its effect on easterly wave disturbances. J. Atmos. Sci., 45, 3102-3136.

— haran air layer on tropical cyclogenesis over the eastern Atlantic. Mon. Wea. Rev., 130, 3100-3128.

Kelley, W. E., and D. R. Mock, 1982: A diagnostic study of upper tropospheric cold lows over the western North Pacific. Mon. Wea. Rev., 110, 471-480.

Kiladis, G. N., H. von Storch, and H. van Loon, 1989: Origin of the South Pacific convergence zone. J. Climate, 2, 1185-1195.

Knapp, K. R., M. C. Kruk, D. H. Levinson, H. J. Diamond, and C. J. Neumann, 2010: The International Best Track Archive for Climate Stewardship (IBTrACS): Unifying tropical cyclone best track data. Bull. Amer. Meteor. Soc., 91, 363-376.

Kousky, V. E., and M. A. Gan, 1981: Upper tropospheric cyclonic vortices in the tropical South Atlantic. Tellus, 33, 538-551.

Krishnamurti, T. N., and V. Wong, 1979: A planetary boundarylayer model for the Somali jet. J. Atmos. Sci., 36, 1895-1907.

Landsea, C. W., and Coauthors, 2004: The Atlantic Hurricane Database Re-Analysis Project: Documentation for the 18511910 alterations and additions to the HURDAT database. Hurricanes and Typhoons: Past, Present and Future, R. J. Murnane and K.-B. Liu, Eds., Columbia University Press, 177-221.

Lazarsfeld, P. F., and N. W. Henry, 1968: Latent Structure Analysis. Houghton Mifflin, 294 pp.

Lee, C. S., R. Edson, and W. M. Gray, 1989: Some large-scale characteristics associated with tropical cyclone development in the North Indian Ocean during FGGE. Mon. Wea. Rev., 117, 407-426.

Leith, C., 1974: Theoretical skill of Monte Carlo forecasts. Mon. Wea. Rev., 102, 409-418.

Leslie, L. M., 1980: Numerical modeling of the summer heat low of Australia. J. Appl. Meteor., 19, 381-387.

Lu, R., C.-S. Ding, H. Ryu, Z. Lin, and H. Dong, 2007: Midlatitude westward propagating disturbances preceding intraseasonal oscillations of convection over the subtropical western North Pacific during summer. Geophys. Res. Lett., 34, L21702, doi:10.1029/2007GL031277.

Martius, O., C. Schwierz, and H. C. Davies, 2007: Breaking waves at the tropopause in the wintertime Northern Hemisphere:
Climatological analyses of the orientation and the theoretical LC1/2 classification. J. Atmos. Sci., 64, 2576-2592.

$\_, \ldots$, and M. Sprenger, 2008: Dynamical tropopause variability and potential vorticity streams in the Northern Hemisphere - A climatological analysis. Adv. Atmos. Sci., 25, 367-379.

McBride, J. L., and T. D. Keenan, 1982: Climatology of tropical cyclone genesis in the Australian region. J. Climatol., 2, 13-33.

McTaggart-Cowan, R., L. F. Bosart, C. A. Davis, E. H. Atallah, J. R. Gyakum, and K. A. Emanuel, 2006: Analysis of Hurricane Catarina (2004). Mon. Wea. Rev., 134, 3029-3053.

— , G. D. Deane, L. F. Bosart, C. A. Davis, and T. J. Galarneau Jr., 2008: Climatology of tropical cyclogenesis in the North Atlantic (1948-2004). Mon. Wea. Rev., 136, 1284-1304.

Molinari, J., D. Knight, M. Dickinson, D. Vollaro, and S. Skubis, 1997: Potential vorticity, easterly waves, and eastern Pacific tropical cyclogenesis. Mon. Wea. Rev., 125, 2699-2708.

Monk, W. H., 1950: On the wind-driven ocean circulation. J. Meteor., 7, 80-93.

Montgomery, M. T., and L. J. Shapiro, 1993: A three-dimensional balance theory for rapidly rotating vortices. J. Atmos. Sci., 50, 3322-3335.

Moore, R. W., and M. T. Montgomery, 2005: Analysis of an idealized, three dimensional diabatic Rossby vortex: A coherent structure of the moist baroclinic atmosphere. J. Atmos. Sci., 62, 2703-2725.

Mozer, J. B., and J. A. Zehnder, 1996: Lee vorticity production by large-scale tropical mountain ranges. Part I: Eastern North Pacific tropical cyclogenesis. J. Atmos. Sci., 53, 521-538.

Nassor, A., and M. R. Jury, 1998: Intra-seasonal climate varibility of Madagascar. Part I: Mean summer conditions. Meteor. Atmos. Phys., 65, 31-41.

Ndarana, T., and D. W. Waugh, 2011: A climatology of Rossby wave breaking on the Southern Hemisphere tropopause. J. Atmos. Sci., 68, 798-811.

Nielsen-Gammon, J. W., and D. A. Gold, 2008: Dynamical diagnosis: A comparison of quasigeostrophy and Ertel potential vorticity. Synoptic-Dynamic Meteorology and Weather Analysis and Forecasting, Meteor. Monogr., No. 33, Amer. Meteor. Soc., 183-202.

Norquist, D. C., E. E. Recker, and R. J. Reed, 1977: The energetics of African wave disturbances as observed during phase III of GATE. Mon. Wea. Rev., 105, 334-342.

Onogi, K., and Coauthors, 2007: The JRA-25 Reanalysis. J. Meteor. Soc. Japan, 85, 369-432.

Palastanga, V., P. J. van Leeuwen, M. W. Schouten, and P. M. de Ruijter, 2007: Flow structure and variability in the subtropical Indian Ocean: Instability of the south Indian Ocean countercurrent. J. Geophys. Res., 112, C01001, doi:10.1029/2005JC003395.

Payne, B., and J. Methven, 2012: The role of baroclinic waves in the initiation of tropical cyclones across the southern Indian Ocean. Atmos. Sci. Lett., 13, 88-94.

Postel, G. A., and M. H. Hitchman, 1999: A climatology of Rossby wave breaking along the subtropical tropopause. J. Atmos. Sci., 56, 359-373.

Ramsawamy, C., 1962: Breaks in the Indian summer monsoon as a phenomenon of interaction between the easterly and the subtropical westerly jet streams. Tellus, 14, 337-349.

Ramsay, H. A., and C. A. Doswell III, 2005: A sensitivity study of hodograph-based methods for estimating supercell motion. Wea. Forecasting, 20, 954-970.

Rao, Y. P., 1976: Southwest monsoon. Meteorological Monograph: Synoptic Meteorology, No. 1, India Meteorological Department, $367 \mathrm{pp}$. 
Reynolds, R. W., N. A. Rayner, T. M. Smith, D. C. Stokes, and W. Wang, 2002: An improved in situ and satellite SST analysis for climate. J. Climate, 15, 1609-1625.

Riehl, H., 1948: On the formation of typhoons. J. Meteor., 5, $247-$ 264.

Ritchie, E. A., and G. J. Holland, 1993: On the interaction of two tropical cyclone scale vortices. II: Discrete vortex patches. Quart. J. Roy. Meteor. Soc., 119, 1363-1379.

— , and — 1997: Scale interactions during the formation of Typhoon Irving. Mon. Wea. Rev., 125, 1377-1396.

__ and _ 1999: Large-scale patterns associated with tropical cyclogenesis in the western Pacific. Mon. Wea. Rev., 127, 2027 2043.

Rogers, R., 2011: Tropical cyclone structure and intensity change. Proc. Seventh Int. Workshop on Tropical Cyclones, WMO/ TD-1561, Saint-Gilles-Les-Bains, La Réunion, France, World Meteorological Organization, 11-17.

Sadler, J. C., 1967: The tropical upper tropospheric trough as a secondary source of typhoons and a primary source of tradewind disturbances. Hawaii Institute of Geophysics Tech. Rep. 67-12, 44 pp.

— 1975: The upper tropospheric circulation over the global tropics. University of Hawaii Department of Meteorology Tech. Rep. UHMET-75-05, 35 pp.

— 1976: A role of the tropical upper tropospheric trough in early season typhoon development. Mon. Wea. Rev., 104, 1266-1278. , 1978: Mid-season typhoon development and intensity change and the tropical upper tropospheric trough. Mon. Wea. Rev., 106, 1137-1152.

Sakamoto, K., and M. Takahashi, 2005: Cut off and weakening processes of an upper cold low. J. Meteor. Soc. Japan, 83, 817 834.

Sato, N., K. Sakamoto, and M. Takahashi, 2005: An air mass with high potential vorticity preceding the formation of the Marcus Convergence Zone. Geophys. Res. Lett., 32, L17801, doi:10.1029/2005GL023572.

Schenkel, B. A., and R. E. Hart, 2012: An examination of tropical cyclone position, intensity and intensity life cycle within atmospheric reanalysis datasets. J. Climate, 25, 3453-3475.

Serra, Y. L., G. N. Kaladis, and M. F. Cronin, 2008: Horizontal and vertical structure of easterly waves in the Pacific ITCZ. J. Atmos. Sci., 65, 1266-1284.
, G. N. Kalidis, and K. I. Hodges, 2010: Tracking and mean structure of easterly waves over the Intra-Americas Sea. J. Climate, 23, 4823-4840.

Shenoi, S. S. C., D. Shankar, and S. R. Shetye, 2002: Differences in heat budgets of the near-surface Arabian Sea and Bay of Bengal: Implications for the summer monsoon. J. Geophys. Res., 107, 3052, doi:10.1029/2000JC000679.

Simmonds, I., K. Keay, and J. A. T. Bye, 2012: Identification and climatology of Southern Hemisphere mobile fronts in a modern reanalysis. J. Climate, 25, 1945-1962.

Simpson, J., E. Ritchie, J. Holland, J. Halverson, and S. Stewart, 1997: Mesoscale interactions in tropical cyclone genesis. Mon. Wea. Rev., 125, 2643-2661.

Stramma, L., 1992: The south Indian Ocean current. J. Phys. Oceanogr., 22, 421-430.

Todd, M., and R. Washington, 1999: Circulation anomalies associated with tropical-temperate troughs in southern Africa and the south West Indian Ocean. Climate Dyn., 15, 937-951.

Trenberth, K. E., 1976: Spatial and temporal variations of the southern oscillation. Quart. J. Roy. Meteor. Soc., 102, 639-653.

Ueda, H., and T. Yasunari, 1996: Maturing process of the summer monsoon over the western North Pacific-A coupled ocean/ atmosphere system. J. Meteor. Soc. Japan, 74, 493-508.

Uppala, S. M., and Coauthors, 2005: The ERA-40 Re-Analysis. Quart. J. Roy. Meteor. Soc., 131, 2961-3012.

Vincent, D. G., 1994: The South Pacific convergence zone (SPCZ): A review. Mon. Wea. Rev., 122, 1949-1970.

— K. K. H. North, R. A. Valesco, and P. G. Ramsey, 1991: Precipitation rates in the tropics based on the $\mathrm{Q}_{1}$-budget method: 1 June 1984-31 May 1987. J. Climate, 4, 1070-1086.

Vincent, E. M., M. Legaine, C. E. Menkes, N. C. Jourdain, P. Marchesiello, and G. Madec, 2009: Interannual variability of the South Pacific Convergence Zone and implications for tropical cyclone genesis. Climate Dyn., 36, 1881-1896.

Wernli, H., and M. Sprenger, 2007: Identification and ERA-15 climatology of potential vorticity streamers and cutoffs near the extratropical tropopause. J. Atmos. Sci., 64, 15691586.

Wu, C.-H., W.-S. Kau, and M.-D. Chou, 2009: Summer monsoon onset in the subtropical western North Pacific. Geophys. Res. Lett., 36, L18810, doi:10.1029/2009GL040168. 